\title{
A radial velocity survey of the Carina Nebula's O-type stars
}

\author{
Megan M. Kiminki ${ }^{\star}$ and Nathan Smith \\ Steward Observatory, University of Arizona, 933 N. Cherry Avenue, Tucson, AZ 85721, USA
}

Accepted 2018 March 14. Received 2018 March 11; in original form 2017 June 17

\begin{abstract}
We have obtained multi-epoch observations of 31 O-type stars in the Carina Nebula using the CHIRON spectrograph on the CTIO/SMARTS $1.5-\mathrm{m}$ telescope. We measure their radial velocities to $1-2 \mathrm{~km} \mathrm{~s}^{-1}$ precision and present new or updated orbital solutions for the binary systems HD 92607, HD 93576, HDE 303312, and HDE 305536. We also compile radial velocities from the literature for 32 additional O-type and evolved massive stars in the region. The combined data set shows a mean heliocentric radial velocity of $0.6 \mathrm{~km} \mathrm{~s}^{-1}$. We calculate a velocity dispersion of $\leq 9.1 \mathrm{~km} \mathrm{~s}^{-1}$, consistent with an unbound, substructured OB association. The $\operatorname{Tr} 14$ cluster shows a marginally significant $5 \mathrm{~km} \mathrm{~s}^{-1}$ radial velocity offset from its neighbour $\operatorname{Tr} 16$, but there are otherwise no correlations between stellar position and velocity. The O-type stars in Cr 228 and the South Pillars region have a lower velocity dispersion than the region as a whole, supporting a model of distributed massive star formation rather than migration from the central clusters. We compare our stellar velocities to the Carina Nebula's molecular gas and find that $\operatorname{Tr} 14$ shows a close kinematic association with the Northern Cloud. In contrast, $\operatorname{Tr} 16$ has accelerated the Southern Cloud by $10-15 \mathrm{~km} \mathrm{~s}^{-1}$, possibly triggering further massive star formation. The expansion of the surrounding $\mathrm{H}$ II region is not symmetric about the O-type stars in radial velocity space, indicating that the ionized gas is constrained by denser material on the far side.
\end{abstract}

Key words: binaries: spectroscopic-stars: early-type-stars: kinematics and dynamicsstars: massive-ISM: evolution - open clusters and associations: individual: Carina Nebula.

\section{INTRODUCTION}

The Carina Nebula is one of the most dramatic star-forming regions in the nearby Galaxy. It is home to more than 70 O-type stars (Smith 2006; Gagné et al. 2011; Alexander et al. 2016), including the defining star of the $\mathrm{O} 2$ spectral type (Walborn et al. 2002a), as well as three late-type hydrogen-rich Wolf-Rayet stars (WNH stars; Smith \& Conti 2008) and the remarkable luminous blue variable (LBV) $\eta$ Carinae (Davidson \& Humphreys 1997). The distribution of these O-type and evolved massive stars is shown in Fig. 1. Roughly half belong to two central clusters, Trumpler (Tr) 14 and $\operatorname{Tr} 16$, while the rest are spread across an area more than $30 \mathrm{pc}$ in diameter. Most of the more distributed stellar population is found in the South Pillars, a region of active star formation to the south of Tr 16 (Smith et al. 2000, 2010b). The combined ionizing radiation and stellar winds from the clustered and distributed massive stars (Smith \& Brooks 2007) has had a major impact on their surroundings, shaping spectacular dust pillars (Smith et al. 2000, 2010b), powering a developing superbubble (Smith et al. 2000), and potentially triggering the observed ongoing star formation (Megeath et al. 1996; Smith et al. 2000; Rathborne et al. 2004; Smith, Stassun \&

^E-mail: mbagley@email.arizona.edu
Bally 2005; Smith et al. 2010b). The feedback-dominated environment of the Carina Nebula is the closest analogue to giant starburst regions like 30 Doradus (e.g. Doran et al. 2013).

Due to the physical extent and complex structure of the Carina Nebula, the relationships between its various clusters and subclusters have been the subject of considerable debate. Photometric and spectroscopic surveys of the stellar populations of $\operatorname{Tr} 14$ and $\operatorname{Tr} 16$ have usually placed the two clusters at a common distance in the range 2.0-3.5 kpc (Feinstein, Marraco \& Muzzio 1973; Thé Bakker \& Antalova 1980a; Thé Bakker \& Tjin A Djie 1980b; Turner \& Moffat 1980; Walborn 1982a,b; Tapia et al. 1988; Cudworth, Martin \& Degioia-Eastwood 1993; Massey \& Johnson 1993; Tovmassian, Hovhannessian \& Epremian 1994; Tapia et al. 2003; Hur, Sung \& Bessell 2012). Other studies concluded that Tr 14 lies 1-2 kpc behind Tr 16 (Walborn 1973; Morrell, Garcia \& Levato 1988) or vice versa (Carraro et al. 2004). The smaller $\operatorname{Tr} 15$ cluster likewise alternated between being considered a foreground (Thé \& Vleeming 1971) or background (Walborn 1973) cluster, until recent X-ray data revealed a stellar bridge between $\operatorname{Tr} 15$ and $\operatorname{Tr} 14$ (Feigelson et al. 2011). The open cluster Collinder (Cr) 228 and the distributed massive stars of the South Pillars region have been variously treated as an extension of Tr 16 (Walborn 1995; Smith \& Brooks 2008), a distinct but same-distance cluster (Herbst 1976; Turner \& Moffat 1980; Tapia et al. 1988; Tovmassian et al. 1994; 


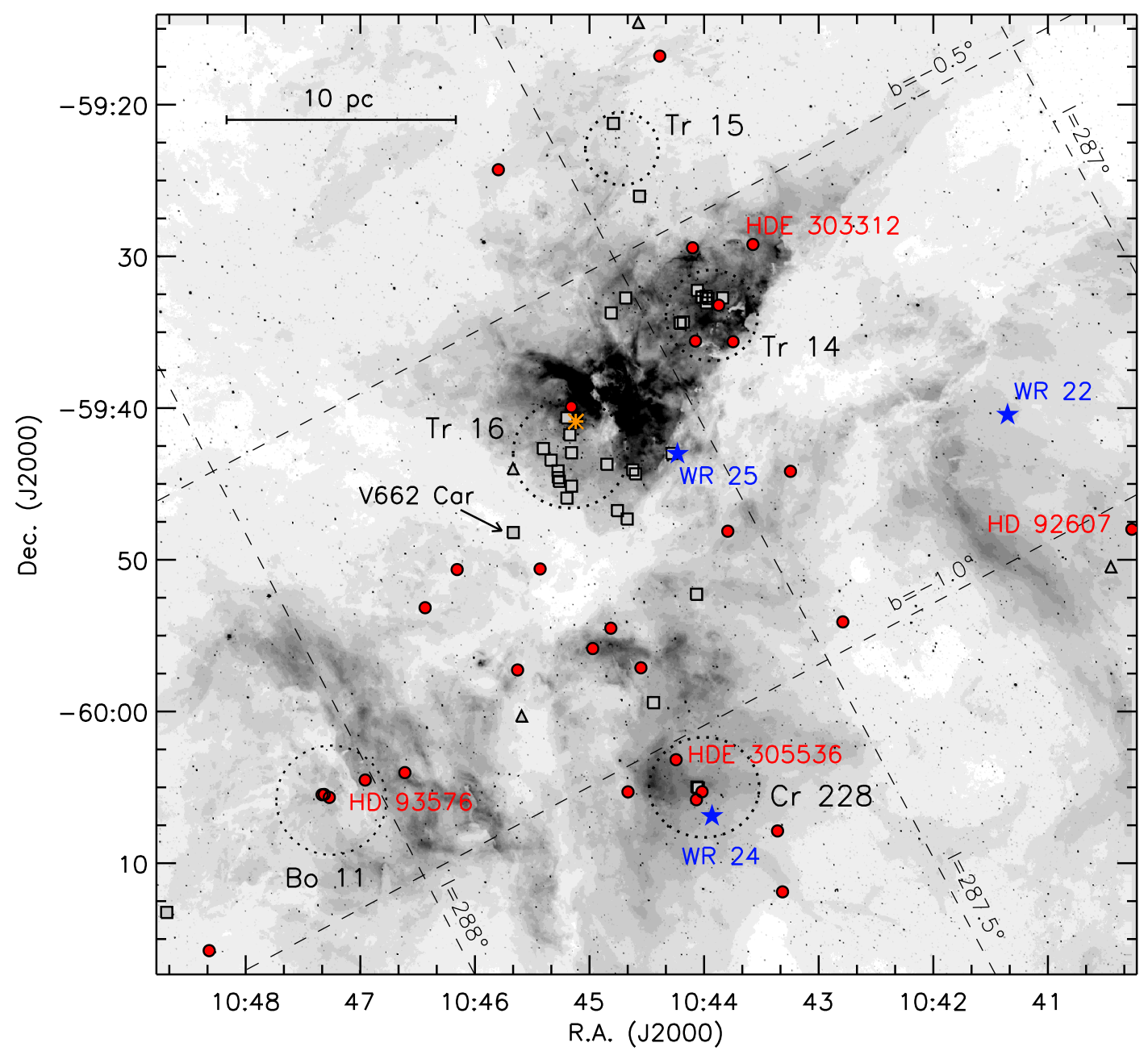

Figure 1. The O-type and evolved massive stars in the Carina Nebula, shown over a ground-based H $\alpha$ image from Smith, Bally \& Walborn (2010a) with Galactic coordinates overlaid for reference. Red circles are O-type stars with new CHIRON observations; we identify by name the four spectroscopic binaries with new orbital solutions (see Section 3). Grey squares are O-type stars in version 3.1 of the GOSC (Maíz Apellániz et al. 2013; Sota et al. 2014) that were not targeted with CHIRON. The V662 Car system (see Section 4.3.2) is highlighted. Grey triangles are additional O-type stars confirmed by Alexander et al. (2016). The three WNH stars are marked with blue stars, and $\eta$ Car is indicated by an orange asterisk. The large dashed circles mark the approximate locations of the major clusters in the region.

Massey et al. 2001, and see also Smith et al. 2010b; Feigelson et al. 2011), or a foreground cluster (Feinstein, Marraco \& Forte 1976; Forte 1978; Carraro \& Patat 2001). The optical studies were complicated by the variable extinction across the region and the unusually high ratio of total-to-selective extinction $R_{\mathrm{V}}$ (Herbst 1976; Forte 1978; Thé et al. 1980a,b; Smith 1987; Tovmassian et al. 1994; Carraro et al. 2004; Mohr-Smith et al. 2017).

Independent of the many spectrophotometric distance studies of the region, measurements of the expansion of $\eta$ Car's Homunculus Nebula have placed that star at a firm distance of $2.3 \mathrm{kpc}$ (Allen \& Hillier 1993; Davidson et al. 2001; Smith 2006). As there is strong evidence that $\eta$ Car is a member of Tr 16 (Walborn \& Liller 1977; Allen 1979) and gas pillars across the Carina Nebula complex show the influence of Tr 16's feedback (Smith et al. 2000, 2010b), a general consensus has arisen that $\operatorname{Tr} 14, \operatorname{Tr} 16, \mathrm{Cr} 228$, and the rest of the stars in the Carina Nebula belong to a single massive association at $2.3 \mathrm{kpc}$ (e.g. Smith \& Brooks 2008, although see Hur et al. 2012). Preliminary results from Gaia Data Release 1 (DR1; Gaia Collaboration et al. 2016a,b; Lindegren et al. 2016), which includes 43 of the Carina Nebula's O-type stars, support this interpretation. The inferred parallax-based distances, as computed by Astraatmadja \& Bailer-Jones (2016) using a prior based on the distribution of stars in the Milky Way, are distributed unimodally around $\sim 2 \mathrm{kpc}$ (Smith \& Stassun 2017).

Even with the spatial link between the Carina Nebula's components now relatively secure, the formation of its distributed massive population remains somewhat uncertain. The O-type stars currently seen among the South Pillars may have been born there, a possible example of an $\mathrm{OB}$ association forming through distributed, hierarchical star formation (e.g. Efremov \& Elmegreen 1998; Clark et al. 2005). But no massive protostars have been detected among the forming stellar population in the South Pillars (Gaczkowski et al. 2013), and Preibisch et al. (2011) argue that the dense gas clouds in the region are not massive enough to support further massive star formation. If the distributed O-type stars did not form in situ, they may have migrated out from Tr 16 either through the classic expansion of a cluster after gas dispersal (Tutukov 1978; Hills 1980; Lada \& Lada 1991, 2003) or as the result of cluster-cluster interaction (Gieles 2013) between $\operatorname{Tr} 14$ and Tr 16. Kiminki et al. (2017) did not see any such outward migration in the local proper 
motions of bow-shock-associated massive stars in the South Pillars, but their sample size was limited.

In this paper, we explore the relationships between the components of the Carina Nebula and the origins of its massive star populations through a survey of the radial velocities (RVs) of its O-type and evolved massive stars. These stellar RVs can be compared to the kinematics of the Carina Nebula's H II regions (e.g. Damiani et al. 2016) and molecular gas (e.g. Rebolledo et al. 2016), allowing a direct assessment of the impact of massive star feedback on the interstellar medium. The RVs of massive stars can be strongly affected by stellar binarity (e.g. Gieles, Sana \& Portegies Zwart 2010), but the effects can be constrained with multi-epoch observations.

The paper is organized as follows: In Section 2, we describe our multi-epoch spectroscopic observing campaign and our RV measurements, and summarize the additional data compiled from the literature. In Section 3, we discuss the O-type stars with variable $\mathrm{RVs}$ and present orbital solutions for four spectroscopic binary systems. Section 4 discusses the RV distributions of the various O-star populations in the Carina Nebula and compares the observed stellar kinematics to the motions of the region's molecular and ionized gas. Our conclusions are summarized in Section 5. Projected distances between sources are given using the $\eta$ Car distance of $2.3 \mathrm{kpc}$.

\section{OBSERVATIONS AND DATA ANALYSIS}

\subsection{Target selection}

Our list of O-type stars in the Carina Nebula is drawn from version 3.1 of the Galactic O-Star Catalog (GOSC; Maíz Apellániz et al. 2013; Sota et al. 2014). The GOSC v3.1 lists 68 objects with right ascensions between 10:40:00 and 10:49:00 and declinations between $-60: 20: 00$ and $-59: 10: 00$, including the Of/WNH system WR 25. An additional four O-type systems in this coordinate range, originally suspected on the basis of their X-ray emission (Povich et al. 2011b), were recently spectroscopically confirmed by Alexander et al. (2016). Adding $\eta$ Car and the remaining two WNH stars brings the total number of known systems with O-type and evolved massive primaries to 75 . These 75 systems are shown in Fig. 1 .

We selected 31 of these systems for new spectroscopic observations. In choosing targets, we prioritized stars that had zero or few prior RV measurements, or whose only existing RV data had high uncertainties. Most of our target stars are thus outside of $\mathrm{Tr}$ 14 and $\operatorname{Tr} 16$, as those clusters have been the targets of multiple spectroscopic campaigns and binary fraction analyses (Penny et al. 1993; García et al. 1998; Albacete Colombo et al. 2001, 2002; Morrell et al. 2001; Rauw et al. 2001, 2009; Nazé et al. 2005; Niemela et al. 2006). We also prioritized sources brighter than $V=11 \mathrm{mag}$.

\subsection{Spectroscopy}

We obtained high-resolution spectra of our 31 target stars with the CHIRON echelle spectrograph (Tokovinin et al. 2013) on the CTIO 1.5-m telescope operated by the SMARTS Consortium. Observations were taken in queue operation using the fibre mode configuration, which provides a resolution of $R \sim 25,000$ over a wavelength range of 4100-8900 A. All 31 stars were observed 2-4 times each from November to December 2014. Observations of a given star were spaced 7-14 d apart to minimize the chances of catching a short-period binary at the same phase, as there is a relative lack of massive binaries in this period range (Kiminki \& Kobulnicky 2012; Kobulnicky et al. 2014). Follow-up observations of eight stars showing possible RV variations were obtained in October 2015-January 2016. Throughout our observing campaign, exposure times were 60-1800 s, designed to achieve signal-to-noise (S/N) ratios of 50-70 at $5500 \AA$. The resulting $\mathrm{S} / \mathrm{N}$ ratios ranged from 30 to 150 with a median of 77 . ThAr calibration spectra were taken before moving the telescope after observing each star; bias and flat-field observations were taken at the beginning and end of each night as part of CHIRON's standard queue observing protocol.

Data were reduced in IRAF $^{1}$ using standard procedures, including bias subtraction, flat-fielding, cosmic ray correction, and extraction of 74 echelle orders. Wavelength calibration was performed using the corresponding ThAr spectrum for each star, and wavelengths were corrected to a heliocentric frame. The wavelength scale of each order is good to an rms of $0.01 \AA\left(\sim 0.6 \mathrm{~km} \mathrm{~s}^{-1}\right.$ at $\left.5000 \AA\right)$. Echelle orders were continuum-normalized before being combined into a single spectrum for each exposure. A representative sample of reduced spectra is shown in Fig. 2, focusing on a wavelength region that covers most of the lines used for RV measurement (see Section 2.3).

\subsection{New radial velocities}

We adapt the method of Sana et al. (2013) for measuring stellar RVs, performing Gaussian fits to a set of He lines and fitting all lines and epochs for a given star simultaneously. The final fit forces all lines at a given epoch to have the same RV, and assumes that the width and amplitude of a given spectral line are constant across epochs. The uncertainties on initial, single-line fits are used to weight each line in the final overall fit. We use the IDL curve-fitting package MPFIT (Markwardt 2009).

Like Sana et al. (2013), we fit to the He absorption lines for two reasons. First, these lines are present across all $\mathrm{O}$ subtypes (unlike most metal lines), allowing us to apply a consistent approach to our full sample of observed stars. Second, the He lines are relatively less sensitive to wind effects compared to the hydrogen Balmer series (Bohannan \& Garmany 1978; see also discussion in Sana et al. 2013). Initially, we fit $\mathrm{He}_{\mathrm{I}} \lambda \lambda 4387,4471,4713,4922,5015,5876$, 6678,7065 , and He II $\lambda \lambda 4541,4686$. The rest wavelengths adopted for these lines are given in Table 1. After the first round of fits, we compared the RVs from individual line fits to the RVs from fitting all lines at once. The triplet blend $\mathrm{He}_{\mathrm{I}} \lambda 4471$ was systematically blueshifted by $\sim 10 \mathrm{~km} \mathrm{~s}^{-1}$ relative to the overall results, and the singlet line $\mathrm{He}$ I $\lambda 6678$ was systematically redshifted by a similar amount. We therefore removed those two lines and refit all epochs of the observed stars. We did not see a systematic offset in the RVs of $\mathrm{He}$ II $\lambda 4686$, likely because the majority of our targets are mid- to late-type main-sequence $\mathrm{O}$ stars, in which this line is not strongly affected by winds (Sana et al. 2013). Wind emission in He II $\lambda 4686$ is apparent in the spectra of the supergiant HD 93632 (see Fig. 2), and we excluded this line in that star's final fit. On a star-by-star basis, we also excluded other lines that were affected by emission from stellar winds or from the surrounding $\mathrm{H}_{\text {II }}$ region, as well as lines that were not present above the noise level of the continuum.

\footnotetext{
${ }^{1}$ IRAF is distributed by the National Optical Astronomy Observatory, which is operated by the Association of Universities for Research in Astronomy (AURA) under a cooperative agreement with the National Science Foundation.
} 


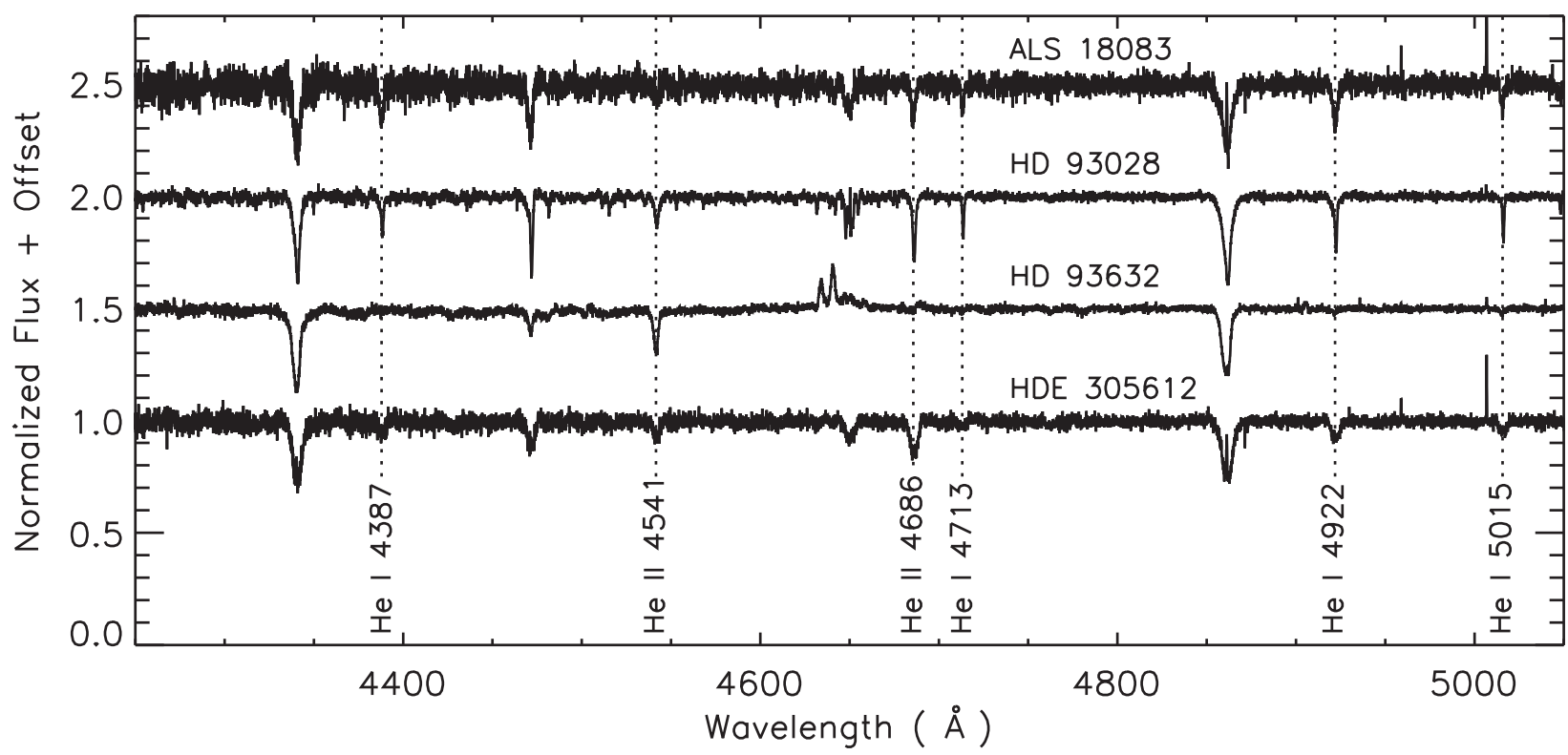

Figure 2. Continuum-normalized CHIRON spectra of four O-type stars in our observed sample, illustrating the range of $\mathrm{S} / \mathrm{N}$ achieved in the wavelength range 4250-5050 Å. Stellar RVs were measured using fits to the marked He I and He II absorption lines and He I $\lambda \lambda 5876$, 7065, where present without emission components.

Table 1. Rest wavelengths used to compute RVs.

\begin{tabular}{|c|c|c|}
\hline Line & Wavelength $(\AA)$ & Reference $^{a}$ \\
\hline $\mathrm{He}_{\mathrm{I}} \lambda 4387$ & 4387.9296 & NIST \\
\hline $\mathrm{He}_{\mathrm{I}} \lambda 4471^{b}$ & 4471.4802 & NIST \\
\hline Не ІІ $\lambda 4541$ & 4541.591 & $\mathrm{PvH}$ \\
\hline Не ІІ $\lambda 4686$ & 4685.71 & $\mathrm{PvH}$ \\
\hline $\mathrm{He}_{\mathrm{I}} \lambda 4713$ & 4713.1457 & NIST \\
\hline Не I $\lambda 4922$ & 4921.9313 & NIST \\
\hline Не г $\lambda 5015$ & 5015.6783 & NIST \\
\hline $\mathrm{He}_{\mathrm{I}} \lambda 5876$ & 5875.621 & NIST \\
\hline $\mathrm{He}_{\mathrm{I}} \lambda 6678^{b}$ & 6678.151 & NIST \\
\hline $\mathrm{He}_{\mathrm{I}} \lambda 7065$ & 7065.190 & NIST \\
\hline
\end{tabular}

$\overline{{ }^{a} \text { NIST }=\text { NIST Atomic Spectra Database v5.4 }}$

(Kramida et al. 2016);

$\mathrm{PvH}=$ Peter van Hoof's Atomic Line List, v2.04

(http://www.pa.uky.edu/ peter/atomic/).

${ }^{b}$ Excluded from final fits.

With these exclusions, an average of seven absorption lines were used to fit the RVs of each star. The measured RVs for each star at each epoch are given in Table 2. The median uncertainty in RV for the single-lined spectra is $1.2 \mathrm{~km} \mathrm{~s}^{-1}$.

One of our observed sources, HD 92607, is a double-lined spectroscopic binary (SB2), first identified by Sexton et al. (2015). We adapted our RV measurement procedure to fit a double Gaussian at all epochs. This process required several steps: First, we fit He I $\lambda 5876$ for the six epochs in which the components were clearly separated. Then, we fixed the width and amplitude of the He I $\lambda 5876$ components and fit to the six epochs with blended components. These fits gave us initial estimates for the RVs at all epochs, which we used as the starting point for fits to $\mathrm{He}_{\mathrm{I}} \lambda \lambda 4922,5015$, and $\mathrm{He}$ II 24686. Again, we fit these lines in the well-separated epochs first, then fixed widths and amplitudes for fits to the blended epochs. The final fit was to all four lines at all epochs. Because the two
Table 2. Heliocentric RVs measured from CHIRON observations of Otype stars in the Carina Nebula. Dates are given for the midpoints of the exposures. This table is available in its entirety as Supporting Information to the online version of this article. A portion is shown here for guidance regarding its form and content.

\begin{tabular}{lrrccc}
\hline Name & $\begin{array}{c}\text { HJD } \\
-2400000\end{array}$ & $\begin{array}{c}\mathrm{RV} 1 \\
\left(\mathrm{~km} \mathrm{~s}^{-1}\right)\end{array}$ & $\begin{array}{c}\sigma(\mathrm{RV} 1) \\
\left(\mathrm{km} \mathrm{s}^{-1}\right)\end{array}$ & $\begin{array}{c}\mathrm{RV} 2 \\
\left(\mathrm{~km} \mathrm{~s}^{-1}\right)\end{array}$ & $\begin{array}{c}\sigma(\mathrm{RV} 2) \\
\left(\mathrm{km} \mathrm{s}^{-1}\right)\end{array}$ \\
\hline ALS 15204 & 56987.814 & -60.0 & 4.7 & - & - \\
ALS 15204 & 56994.850 & 1.0 & 2.3 & - & - \\
ALS 15204 & 57003.836 & 43.1 & 2.0 & - & - \\
ALS 15206 & 56992.858 & 24.5 & 0.4 & - & - \\
ALS 15206 & 57000.851 & 29.8 & 0.5 & - & - \\
ALS 15206 & 57008.788 & 34.1 & 0.3 & - & - \\
\hline
\end{tabular}

components of HD 92607 are of similar spectral type, several iterations of line fitting and orbital analysis (see Section 3.1) were required to confidently identify which line component was associated with which star at a given epoch. The median uncertainty on the RVs of the components of HD 92607 is $7.4 \mathrm{~km} \mathrm{~s}^{-1}$, and ranges as high as $30-50 \mathrm{~km} \mathrm{~s}^{-1}$ in epochs where the lines are highly blended.

As a check on our wavelength calibration, we obtained CHIRON spectra of the RV standard stars HIP 51722 (spectral type F7-8 V) and HIP 53719 (K1-2 IV), both from the catalogue of Soubiran et al. (2013), in November 2014. As the spectra of these standard stars do not have He I lines, their RVs could not be measured by the same method used for our science spectra. Instead, we measured their RVs by cross-correlation with high-resolution stellar spectra from Coelho (2014), using the IRAF task fxcor. The measured $\mathrm{RV}$ s of both standard stars agree with their expected values within $0.4 \mathrm{~km} \mathrm{~s}^{-1}$. 
Table 3. Mean or systemic heliocentric RVs adopted from the literature.

\begin{tabular}{|c|c|c|c|c|}
\hline Name & $\begin{array}{l}\overline{\mathrm{RV}} \text { or } \gamma \\
\left(\mathrm{km} \mathrm{s}^{-1}\right)\end{array}$ & $\begin{array}{c}\sigma(\overline{\mathrm{RV}}) \\
\left(\mathrm{km} \mathrm{s}^{-1}\right)\end{array}$ & $\begin{array}{c}\text { Spectral flag from } \\
\text { Chini et al. (2012) }\end{array}$ & References \\
\hline ALS 15229 & 14.7 & 3.5 & - & García et al. (1998) \\
\hline CPD-58 2611 & 4.7 & 5.0 & $\mathrm{C}^{b}$ & Penny et al. (1993); García et al. (1998) \\
\hline CPD-58 2620 & -0.6 & 5.8 & SB1 & Penny et al. (1993); García et al. (1998) \\
\hline CPD-59 2591 & -2.9 & 10. & - & Huang \& Gies (2006) \\
\hline CPD-59 2624 & 13.5 & 10. & - & Huang \& Gies (2006); SB2 in Alexander et al. (2016) \\
\hline CPD-59 2626 & -22.7 & 10. & - & Huang \& Gies (2006) \\
\hline CPD-59 2627 & -15.9 & 10 . & - & Huang \& Gies (2006) \\
\hline CPD-59 2629 & -8.8 & 12.8 & - & SB1 in Williams et al. (2011) \\
\hline CPD-59 2635 & 0 . & 1. & SB2 & SB2 solution from Albacete Colombo et al. (2001) \\
\hline CPD-59 2636 & 4.0 & 5.7 & SB2 & Quadruple system; Albacete Colombo et al. (2002) \\
\hline CPD-59 2641 & -4.5 & 1.8 & SB2 & SB2 solution from Rauw et al. (2009) \\
\hline$\eta$ Carinae & -8.1 & 1. & - & Smith (2004) \\
\hline HD 93128 & 5.0 & 5.3 & $\mathrm{C}^{b}$ & Penny et al. (1993); García et al. (1998) \\
\hline HD 93129A & -10.1 & 18.7 & $\mathrm{C}^{b}$ & Conti et al. (1979); Penny et al. (1993) \\
\hline HD 93129B & 7.1 & 2.2 & - & Penny et al. (1993) \\
\hline HD 93130 & 59.9 & 3.1 & SB2 & Conti et al. (1977) \\
\hline HD 93160 & -12.9 & 17.9 & SB1 & Thackeray, Tritton \& Walker (1973); Conti, Leep \& Lorre (1977) \\
\hline HD 93161A & 1.1 & 3.6 & SB2 & SB2 data from Nazé et al. (2005) \\
\hline HD 93161B & -25.4 & 28.4 & - & Nazé et al. (2005) \\
\hline HD 93204 & 8.5 & 3.8 & $\mathrm{C}$ & Conti et al. (1977) \\
\hline HD 93205 & -2.9 & 0.9 & SB2 & SB2 solution from Morrell et al. (2001) \\
\hline HD 93250 & -5.2 & 7.6 & $\mathrm{C}^{b}$ & Thackeray et al. (1973); Rauw et al. (2009); Williams et al. (2011) \\
\hline HD 93343 & -2.8 & 54.4 & SB2 & SB2 data from Rauw et al. (2009) \\
\hline HD 93403 & -14.2 & 5.4 & SB2 & SB2 solution from Rauw et al. (2000) \\
\hline HD 93843 & -9.9 & 0.4 & $\mathrm{C}$ & Feast et al. (1957); Conti et al. (1977) \\
\hline QZ Car & -8.7 & 15.5 & SB2 & Quadruple system; Morrison \& Conti (1980); Mayer et al. (2001) \\
\hline Trumpler $14-9$ & 5.6 & 3.8 & - & Penny et al. (1993); García et al. (1998) \\
\hline V572 Car & -3.4 & 3.7 & SB2 & Triple system; Rauw et al. (2001) \\
\hline V573 Car & -5 & 4. & - & SB2 solution from Solivella \& Niemela (1999) \\
\hline V662 Car & -15 & 2. & - & SB2 solution from Niemela et al. (2006) \\
\hline WR $22^{c}$ & -27.5 & - & - & SB1 solution from Schweickhardt et al. (1999) \\
\hline $\mathrm{WR} 24^{c}$ & -34.0 & 13.3 & - & Conti et al. (1979) \\
\hline WR $25^{c}$ & -34.6 & 0.5 & - & SB1 solution from Gamen et al. (2006) \\
\hline
\end{tabular}

${ }^{a} \mathrm{C}=$ constant; $\mathrm{SB} 1=$ single-lined spectroscopic binary; SB2 = double-lined spectroscopic binary.

${ }^{b}$ Although classified as constant in Chini et al. (2012), these stars meet our criterion for significant RV variability $\left(P\left(\chi^{2}, v\right)<0.01\right.$; see Section 3$)$ and have RV amplitudes $>20 \mathrm{~km} \mathrm{~s}^{-1}$.

${ }^{c}$ Reported RVs are from N IV $\lambda 4058$ emission; see discussion in Section 2.4.

\subsection{Radial velocities from the literature}

Where available, we also compiled heliocentric RVs from the literature for the Carina Nebula's O-type and evolved massive stars. The adopted RVs and associated uncertainties for stars not observed with CHIRON are given in Table 3 along with the corresponding references. Nearly all of these RVs were measured from various subsets of $\mathrm{He}$ absorption lines, often in conjunction with metal and/or hydrogen Balmer lines. The exceptions are: $\eta$ Car, where the systemic $\mathrm{RV}$ was derived from $\mathrm{H}_{2}$ emission from the Homunculus (Smith 2004); the three WNH stars, discussed further below; and the Conti, Niemela \& Walborn (1979) RVs for the Of supergiant HD 93129A, where we use RVs from the 'Group 1' narrow metal lines. Where a system is a spectroscopic binary with a published orbital solution, we give the systemic velocity; for other sources with observations at multiple epochs, we give the weighted mean of the available velocities. For visual binaries that are resolvable at the few-arcsecond level (e.g. HD 93161AB), we use only data that specify which component was observed. We also note whether systems were classified as constant-RV or binary in the multiplicity survey of Chini et al. (2012). Additional notes on the spectroscopic multiplicity of a system are given in the references column. We also incorporate literature data for the following CHIRON targets: ALS 15206 (Huang \& Gies 2006), ALS 15207 (García et al. 1998), HD 93028 (Feast, Thackeray \& Wesselink 1957; Conti, Leep \& Lorre 1977), HDE 303308 (Conti et al. 1977), HDE 305518 (Huang \& Gies 2006), and HDE 305619 (Humphreys 1973). In no case does the inclusion of these sources' literature RVs shift their overall weighted mean RV by more than $1.5 \mathrm{~km} \mathrm{~s}^{-1}$.

As mentioned above, the three WNH stars are a special case. Like all Wolf-Rayet stars, these sources have strong stellar winds, with their spectral features formed at various depths within those winds (e.g. Crowther 2007). Consequently, the observed RVs vary greatly depending on which lines are measured. Relative to the stars' systemic velocities, the hydrogen Balmer and He II absorption lines of Wolf-Rayet spectra tend to be highly blueshifted (Moffat 1978; Moffat \& Seggewiss 1978; Conti et al. 1979; Massey \& Conti 1981; Rauw et al. 1996), while their He II emission lines tend to be highly redshifted (Moffat 1978; Moffat \& Seggewiss 1978; Massey 1980; Niemela \& Moffat 1982; Schweickhardt et al. 1999; Schnurr et al. 2008). In WN and WNH spectra, certain narrow $\mathrm{N}$ III, $\mathrm{N}_{\text {IV }}$, and $\mathrm{Si}$ IV emission lines are thought to be 
relatively better tracers of the true stellar RVs (e.g. Moffat 1978; Moffat \& Seggewiss 1978, 1979; Conti et al. 1979). For the Carina Nebula's WNH stars, we adopt RVs measured from N IV $\lambda 4058$ emission.

However, it is clear that the adopted RVs for the WNH stars are still affected by winds. Using the spectral features of the Otype companion to WR 22, Rauw et al. (1996) calculated the true systemic velocity of the binary to be $21.3 \pm 7.0 \mathrm{~km} \mathrm{~s}^{-1}$, while Schweickhardt et al. (1999) report $\approx 0 \pm 15 \mathrm{~km} \mathrm{~s}^{-1}$ for the same source. The N IV $\lambda 4058$ line in the spectrum of WR 22 is thus blueshifted by $\sim 25-50 \mathrm{~km} \mathrm{~s}^{-1}$. In other $\mathrm{WN}+\mathrm{O}$ binaries, this line shows relative blueshifts of $0-80 \mathrm{~km} \mathrm{~s}^{-1}$ (Niemela, Massey \& Conti 1980; Niemela \& Moffat 1982; Collado, Gamen \& Barbá 2013; Collado et al. 2015; Munoz et al. 2017). Because the exact magnitude of the shift is uncertain and seems to vary by star, we do not attempt to correct the $\mathrm{N}$ IV $\lambda 4058 \mathrm{RVs}$ for wind effects, and we consider the three WNH stars as a separate group in our analysis. Note that although $\eta \mathrm{Car}$ is also an evolved massive star with a high mass-loss rate, its RV was measured by a different method (Smith 2004) and it does not suffer from the same bias.

In compiling literature RVs for Table 3, we found that the RVs in Levato et al. (1990, 1991a,b) are notably discrepant with later observations, including our own. These three studies measured RVs from an unspecified subset of hydrogen Balmer and He absorption lines for a total of 92 candidate members of $\mathrm{Cr} 228, \operatorname{Tr} 16$, and $\operatorname{Tr}$ 14, using the spectrograph on the 1-m Yale CTIO telescope (see Levato et al. 1986). They computed average cluster RVs of -23 , -26 , and $-29 \mathrm{~km} \mathrm{~s}^{-1}$ for $\mathrm{Cr} 228, \operatorname{Tr} 16$, and $\operatorname{Tr} 14$, respectively. By contrast, the surveys of Penny et al. (1993) and García et al. (1998) found +2.8 and $+6.0 \mathrm{~km} \mathrm{~s}^{-1}$, respectively, for the average RV of Tr 14. Penny et al. (1993) also reported very different results from Levato et al. (1991b), in both RV values and variability, for individual sources in $\operatorname{Tr} 14$. Across the Carina Nebula, we find that the Levato et al. (1990, 1991a,b) data are typically $10-60 \mathrm{~km} \mathrm{~s}^{-1}$ more negative than other literature values for the same stars (see Table 3) and our CHIRON observations. We therefore exclude all data from the Levato et al. studies, except for specific binary orbits as discussed in Section 3.

With the inclusion of the literature data in Table 3, we have RVs for 63 of the 75 O-type and evolved massive stars in the Carina Nebula. Four of the 12 stars without RV data are those that were newly confirmed by Alexander et al. (2016). These four are relatively highly extincted, with a median $A_{\mathrm{V}}$ of 5.7 mag compared to the median $A_{\mathrm{V}}$ of $2.2 \mathrm{mag}$ for the rest of the O-type stars in the region (Gagné et al. 2011; Povich et al. 2011a). Alexander et al. (2016) postulate that one of these, $\mathrm{OBc} 3$, is a coincidentally aligned background star. The other eight stars without RV data are neither systematically more extincted nor systematically fainter at visual wavelengths than the 63 sources with RV data. Some of these eight have been observed as part of the OWN RV survey (Barbá et al. 2010; see Sota et al. 2014; Maíz Apellániz et al. 2016), but currently lack published RVs.

\section{NEW AND UPDATED BINARY ORBITS}

Multiplicity is ubiquitous among O-type stars (Garmany, Conti \& Massey 1980; Mason et al. 2009; Chini et al. 2012; Sana et al. 2012, 2013, 2014; Kobulnicky et al. 2014; Moe \& Di Stefano 2017) and must be considered when determining their systemic RVs. For each of our CHIRON sources, we compute $P\left(\chi^{2}, v\right)$, the probability that the $\chi^{2}$ about the weighted mean would be exceeded by random chance given $v=N_{\mathrm{obs}}-1$ degrees of freedom. Nine of the 31 observed stars display significant $\left(P\left(\chi^{2}, v\right)<0.01\right) \mathrm{RV}$ variations with amplitudes $>20 \mathrm{~km} \mathrm{~s}^{-1}$ in our CHIRON data. As discussed below, we are able to find periods and fit orbital solutions to four of these spectroscopic binaries. Two additional observed sources meet the criteria for significant, high-amplitude variations when their literature RVs are included. One of these sources is HD 93403, a known SB2 with a full orbital solution in Rauw et al. (2000). Our CHIRON observations are in good agreement with the orbit of the primary star, and we adopt the systemic velocity for HD 93403 from the Rauw et al. (2000) solution.

An additional five CHIRON targets display significant $\left(P\left(\chi^{2}\right.\right.$, $v)<0.01)$, but low-amplitude $\left(\Delta \mathrm{RV}<20 \mathrm{~km} \mathrm{~s}^{-1}\right) \mathrm{RV}$ variations. These stars include HDE 305619, which was flagged as an SB1 by Chini et al. (2012) but has minimal published RV data. The other four stars may have undetected long-period companions or may be showing photospheric variability (e.g. Garmany, Conti \& Massey 1980; Gies \& Bolton 1986; Fullerton, Gies \& Bolton 1996; Ritchie et al. 2009; Martins et al. 2015).

We search for orbital solutions for the stars with significant, high-amplitude RV variations and $N_{\text {obs }} \geq 7$ using the IDL package RVFIT (Iglesias-Marzoa, López-Morales \& Jesús Arévalo Morales 2015). Spectroscopic binary orbits are defined by the following parameters: $P$ (orbital period), $e$ (eccentricity), $\gamma$ (systemic velocity), $\omega$ (argument of periastron), $T_{\mathrm{p}}$ (epoch of periastron), $K_{1}$ (primary semi-amplitude), and, where applicable, $K_{2}$ (secondary semiamplitude). RVFIT uses an adaptive simulated annealing method to find the global $\chi^{2}$ minimum in the six- or seven-dimensional parameter space. Unlike some orbital fitting codes, RVFIT does not use an initial estimate of the period. In four cases, described in further detail below, RVFIT converged on orbital solutions with well-fitted phase-folded velocity curves. Parameter uncertainties were computed using RVFIT's Markov Chain Monte Carlo (MCMC) algorithm (Iglesias-Marzoa et al. 2015) with $10^{5}$ points. The MCMC provides the probability distribution of each parameter marginalized over all other parameters. For our data, these marginalized histograms are well approximated by Gaussian distributions and we take their standard deviations as the $1 \sigma$ uncertainties on the orbital parameters.

During our initial analysis of the variable-RV sources, we computed the frequency power spectrum of each source using A. W. Fullerton's IDL implementation of the one-dimensional CLEAN algorithm (Roberts, Lehar \& Dreher 1987). We confirm the periods returned by RVFIT by checking against these periodograms, which are presented in Appendix A.

\subsection{HD 92607}

HD 92607 was first noted as an SB2 by Sexton et al. (2015), who classified its components as $08.5 \mathrm{~V}+\mathrm{O} 9 \mathrm{~V}$. It is on the far western side of the Carina Nebula, approximately $25 \mathrm{pc}$ from Tr 14 and 16 (see Fig. 1). The lower mass stellar population in this area, by the edge of the Carina Nebula's northern molecular cloud, is relatively young ( $<1 \mathrm{Myr}$; Kumar et al. 2014). HD 92607 is associated with an extended arc of $24 \mu \mathrm{m}$ emission, suggestive of a stellar wind bow shock, that points to the south-east (Sexton et al. 2015).

We obtained 12 CHIRON spectra of HD 92607 in 2014-2015. As described in Section 2.3, we adapted our RV-fitting procedure to fit two Gaussian profiles to He I $\lambda \lambda 4922,5015,5876$, and He II $\lambda 4686$ across all 12 epochs, using fits to the well-separated epochs to fix the widths and amplitudes of the lines. We used RVFIT to fit 


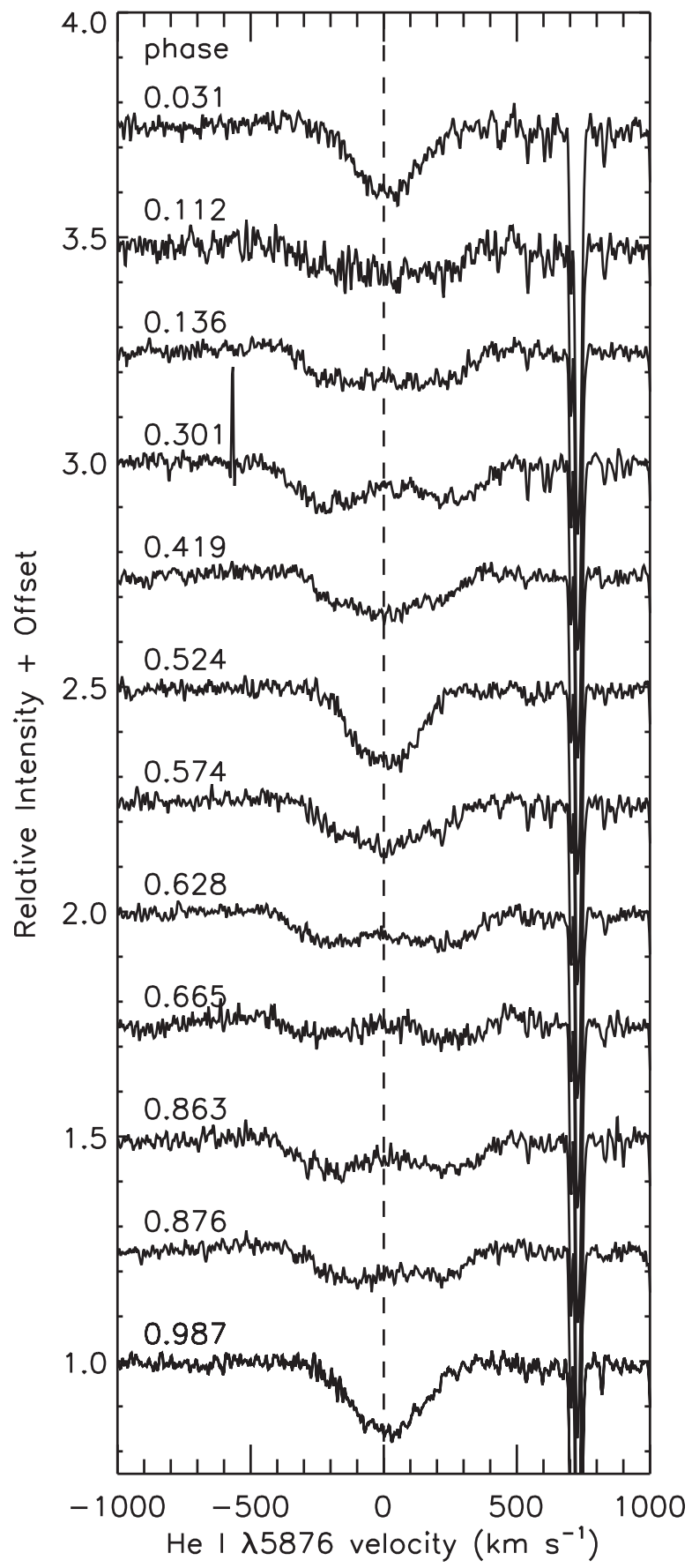

Figure 3. He I $\lambda 5876$ in velocity space and in order of phase for CHIRON observations of the SB2 HD 92607, for an orbital period of $3.6993 \mathrm{~d}$. The narrow absorption line on the right is interstellar Na I $\lambda 5890$.

an orbit to the primary and secondary RVs simultaneously. We fit a common apparent systemic velocity to both components. ${ }^{2}$

The best-fitting period for HD 92607 is $3.6993 \pm 0.0001$ d. Fig. 3 shows the progression of $\mathrm{He}$ I $\lambda 5876$ over this period. After our initial orbital fit converged on an eccentricity of zero, we fixed $e=0$ for further fitting, allowing RVFIT to treat $\omega$ and $T_{\mathrm{p}}$ (which are ill de-

\footnotetext{
${ }^{2}$ We used an $F$-test to compare our single- $\gamma$ solution to the best-fitting orbit computed with different $\gamma$ values for each component. The latter did not provide a significant improvement in fit $(p=0.27)$.
}

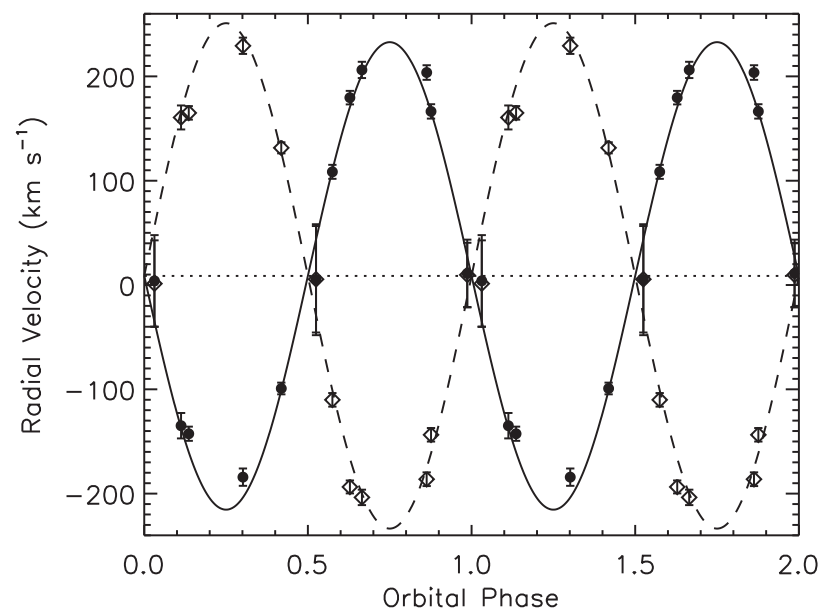

Figure 4. RV curve and orbital solution for the SB2 HD 92607 at the best-fitting orbital period of $3.6993 \mathrm{~d}$. Filled circles and open diamonds correspond to the primary and secondary components, respectively. The dotted line marks the systemic velocity.

fined at very low eccentricities) in a consistent manner (see IglesiasMarzoa et al. 2015). The final best-fitting orbital solution is shown in Fig. 4, and the orbital parameters are listed in Table 4. In the bestfitting orbit, the minimum masses of the primary and secondary stars are $M_{1} \sin ^{3} i=20.2 \pm 0.6 \mathrm{M}_{\odot}$ and $M_{2} \sin ^{3} i=18.7 \pm 0.6 \mathrm{M}_{\odot}$, respectively, giving a mass ratio of $q=0.93 \pm 0.04$. But their spectral types of O8.5 V and O9 V (Sexton et al. 2015), according to the 'observational' scale of Martins, Schaerer \& Hillier (2005), have somewhat lower expected masses of $M_{1}=18.8 \mathrm{M}_{\odot}$ and $M_{2}=17.1 \mathrm{M}_{\odot}$. The apparent discrepancy could be the result of the normal uncertainties on spectral-type classification; for instance, Gagné et al. (2011) list HD 92607 as an O8 V star, although they do not account for its spectroscopic binarity. Or perhaps the components of HD 92607 are slightly older, and hence cooler for their masses, than average main-sequence stars of their types. In any case, the HD 92607 system is likely close to edge-on $\left(i \sim 90^{\circ}\right)$, as a smaller inclination would imply implausibly large masses for its component stars.

\subsection{HD 93576}

HD 93576 is one of five O-type systems in the open cluster Bochum (Bo) 11 in Carina's South Pillars region. Its primary is of type O99.5 IV-V (Sota et al. 2014; Sexton et al. 2015). It is associated with an extended arc of $8 \mu \mathrm{m}$ emission, which points away from Bo 11 and has infrared colours indicative of a stellar wind bow shock (Sexton et al. 2015). Its local proper motion confirms that it is moving away from Bo 11 at $\lesssim 15 \mathrm{~km} \mathrm{~s}^{-1}$; it may have been recently ejected from the cluster (Kiminki et al. 2017).

Levato et al. (1990) first noted periodic RV variations in the spectra of HD 93576, and fit an orbital solution with a period of $2.020 \mathrm{~d}$. We obtained 12 CHIRON observations of this system in 2014-2016. We used RVFIT to compute orbital solutions both with and without the Levato et al. (1990) data. With our CHIRON data alone, we find a period of $1.85201 \mathrm{~d}$; including the Levato et al. (1990) data constrains the period to $1.852102 \pm 0.000002 \mathrm{~d}$. The final best-fitting parameters given in Table 4 and used to plot the orbital solution in Fig. 5 are from the combined data set. As can be seen in Fig. 5, many but not all of the Levato et al. (1990) RVs for this system are $10-20 \mathrm{~km} \mathrm{~s}^{-1}$ blueshifted relative to CHIRON data 
Table 4. Orbital and physical parameters of new and updated binary solutions.

\begin{tabular}{lcccc}
\hline Element & HD 92607 & HD 93576 & HDE 303312 & HDE 305536 \\
\hline$P(\mathrm{~d})$ & $3.6993(0.0001)$ & $1.852102(0.000002)$ & 9.4111 (fixed) & $1.88535(0.00002)$ \\
$e$ & 0.00 (fixed) & $0.075(0.009)$ & 0.58 (fixed) & $0.129(0.008)$ \\
$\gamma\left(\mathrm{km} \mathrm{s}^{-1}\right)$ & $8.8(1.6)$ & $-8.4(0.6)$ & $2.3(0.6)$ & $2.3(0.2)$ \\
$\omega\left({ }^{\circ}\right)$ & 90 (fixed) & $201(9)$ & $192(2)$ & $56(3)$ \\
$T_{\mathrm{p}}(\mathrm{HJD}-2400000)$ & $56965.910(0.009)$ & $57005.94(0.05)$ & $56980.58(0.03)$ & $56975.34(0.02)$ \\
$K_{1}\left(\mathrm{~km} \mathrm{~s}^{-1}\right)$ & $224(3)$ & $85.0(0.8)$ & $71(1)$ & $37.3(0.3)$ \\
$K_{2}\left(\mathrm{~km} \mathrm{~s}^{-1}\right)$ & $242(3)$ & - & - & - \\
$f\left(M_{1}, M_{2}\right)\left(\mathrm{M}_{\odot}\right)$ & - & $0.117(0.004)$ & $0.19(0.01)$ & $0.0099(0.0004)$ \\
$M_{1} \sin ^{3} i\left(\mathrm{M}_{\odot}\right)$ & $20.2(0.6)$ & - & - & - \\
$M_{2} \sin ^{3} i\left(\mathrm{M}_{\odot}\right)$ & $18.7(0.6)$ & - & - & - \\
$a_{1} \sin _{i}\left(\mathrm{R}_{\odot}\right)$ & $16.4(0.3)$ & $3.10(0.03)$ & $10.7(0.2)$ & $1.38(0.01)$ \\
$a_{2} \sin i\left(\mathrm{R}_{\odot}\right)$ & $17.7(0.2)$ & - & - & - \\
$\mathrm{rms}_{1}\left(\mathrm{~km} \mathrm{~s}^{-1}\right)$ & 19.7 & 10.9 & - & - \\
$\mathrm{rms}_{2}\left(\mathrm{~km} \mathrm{~s}^{-1}\right)$ & 23.7 & - & - & - \\
\hline
\end{tabular}

Notes: $1 \sigma$ uncertainties for each quantity are listed in parentheses.

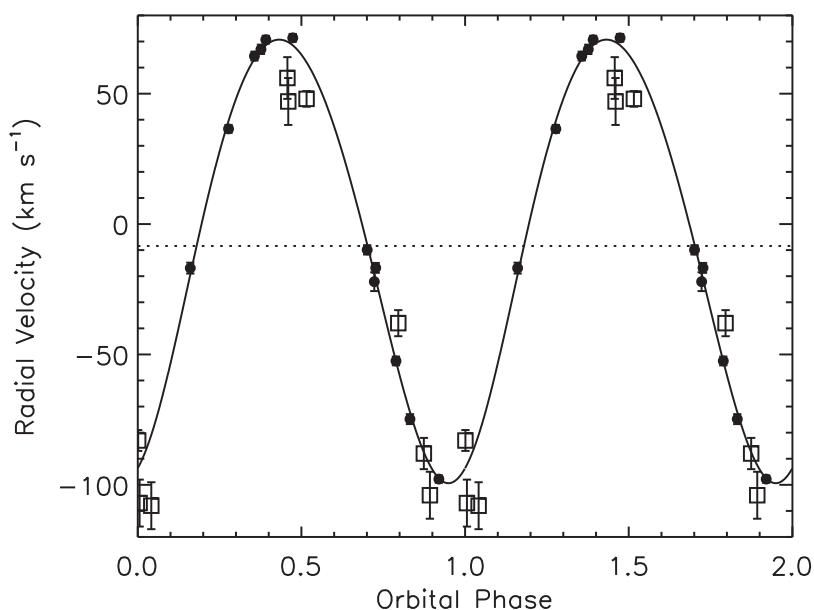

Figure 5. RV curve and orbital solution for the SB1 HD 93576 at the bestfitting orbital period of $1.852102 \mathrm{~d}$. Solid circles are measurements from our CHIRON spectra and open squares are data from Levato et al. (1990). Both data sets were used to compute the orbital solution. The dotted line marks the systemic velocity.

at the same phase. Levato et al. (1990) reported a systemic velocity of $-21 \mathrm{~km} \mathrm{~s}^{-1}$ for this system, while our fit to the combined data set finds a systemic velocity of $-8.4 \mathrm{~km} \mathrm{~s}^{-1}$ and fitting to only our CHIRON data gives a systemic velocity of $-8.2 \mathrm{~km} \mathrm{~s}^{-1}$.

The orbit of HD 93576 is minimally but significantly non-circular, with an eccentricity of $0.075 \pm 0.009$. The resulting mass function is $f\left(M_{1}, M_{2}\right)=0.117 \pm 0.004 \mathrm{M}_{\odot}$. Assuming the primary star has a mass of 16-18 $\mathrm{M}_{\odot}$, as appropriate for its spectral type per Martins et al. (2005), the minimum mass of the secondary star is $\sim 3.7 \mathrm{M}_{\odot}$, roughly the mass of a B8 V star (Drilling \& Landolt 2000).

\subsection{HDE 303312}

HDE 303312 is an O9.7 IV star (Sota et al. 2014) located on the outskirts of $\operatorname{Tr} 14$ (see Fig. 1). There are no RVs for this source in the literature, but it was identified as an eclipsing system by Otero (2006) based on its $V$-band light curve from the All Sky Automated Survey (ASAS; Pojmanski 1997). Otero (2006) report a period of $9.4109 \mathrm{~d}$ and note that it is an 'extremely eccentric' system. We obtained a total of 12 CHIRON observations of HDE 303312 over 2014-2015, and downloaded the available ASAS
$V$-band magnitudes for 2000-2009. In addition to using RVFIT on the velocity data, we use the eclipsing binary modelling programs PHOEBE (Prša \& Zwitter 2005) and NIGHTFALL ${ }^{3}$ to synthesize and compare photometric light curves. We find that the ASAS light curve folds most cleanly at a period of $9.4111 \mathrm{~d}$ rather than $9.4109 \mathrm{~d}$. We therefore adopt $9.4111 \mathrm{~d}$ as the photometric period with an estimated uncertainty of $\pm 0.0002 \mathrm{~d}$.

Initial, unconstrained runs of RVFIT find an RV period between 9.41 and $9.65 \mathrm{~d}$, with an eccentricity of 0.6-0.7. These results confirm that the eclipsing and spectroscopic binaries are the same system, and we subsequently fix the period at $9.4111 \mathrm{~d}$. With all other orbital parameters left free, the best-fitting solution to the RV data has $e=0.67$ and $\omega=188^{\circ}$. Matching the phase separation of the $V$-band eclipses for $e=0.67$ requires $\omega \approx 140^{\circ}$ or $220^{\circ}$, incompatible with the shape and symmetry of the RV curve. We do not see any shifts in eclipse timing over the nine years of ASAS data that would suggest apsidal motion as an explanation for this discrepancy. We therefore iterate between the RV and light curves to converge on values of $e, \omega$, and inclination $i$ that are consistent with the observed data. Our final best fit to the RV curve, presented in Table 4 and plotted in Fig. 6, holds the eccentricity fixed at 0.58 and gives $\omega=192 \pm 2^{\circ}$. The inclination of the system is $80^{\circ}-85^{\circ}$.

This best-fitting orbital solution for HDE 303312 gives a mass function of $f\left(M_{1}, M_{2}\right)=0.19 \pm 0.01 \mathrm{M}_{\odot}$. If we take the mass of the primary star to be $15-18 \mathrm{M}_{\odot}$, based on its spectral type (see Martins et al. 2005), the mass of the secondary star is $4-5 \mathrm{M}_{\odot}$. Assuming the secondary star is on the main sequence, that mass corresponds to a spectral type of B6-7 V (Drilling \& Landolt 2000), which would have an effective temperature of $T_{\text {eff }} \approx 13000-14000 \mathrm{~K}$ (Kenyon \& Hartmann 1995). Unfortunately, the noise in the ASAS data prevents us from putting strong constraints on the relative luminosities or stellar radii of the components. High-S/N, multiwavelength photometry is needed to confirm the characteristics of the secondary star.

\subsection{HDE 305536}

The O9.5 V star (Sota et al. 2014) HDE 305536 is located close to the optical centre of the open cluster Cr 228 (e.g. Wu et al. 2009), a few pc from the WNH star WR 24. Like HD 93576, it is associated

\footnotetext{
${ }^{3}$ http://www.hs.uni-hamburg.de/DE/Ins/Per/Wichmann/Nightfall
} 


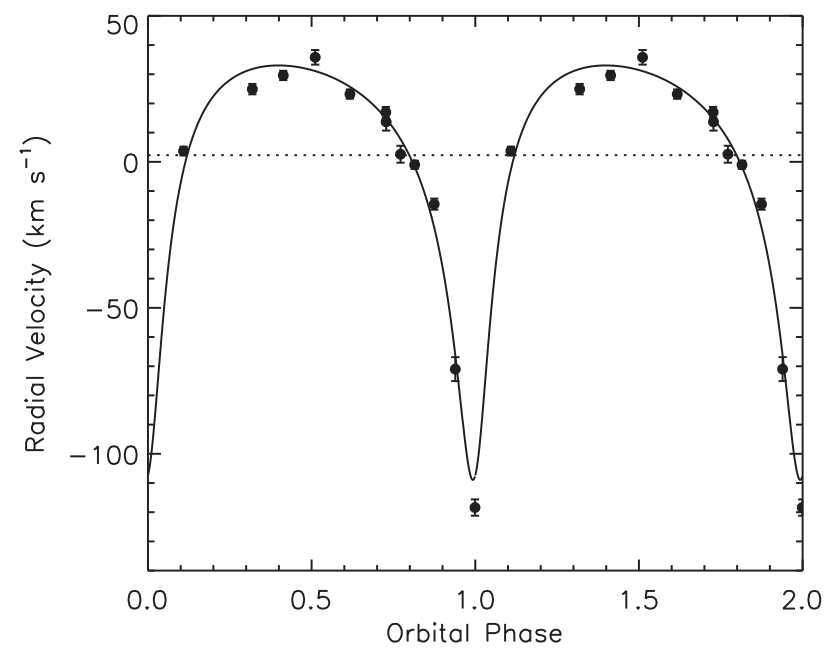

Figure 6. RV curve and orbital solution for the eclipsing SB1 HDE 303312. The solid line shows the best-fitting orbital solution to the RV data with the period and eccentricity fixed at $9.4111 \mathrm{~d}$ and 0.58 , respectively. The dotted line marks the systemic velocity.

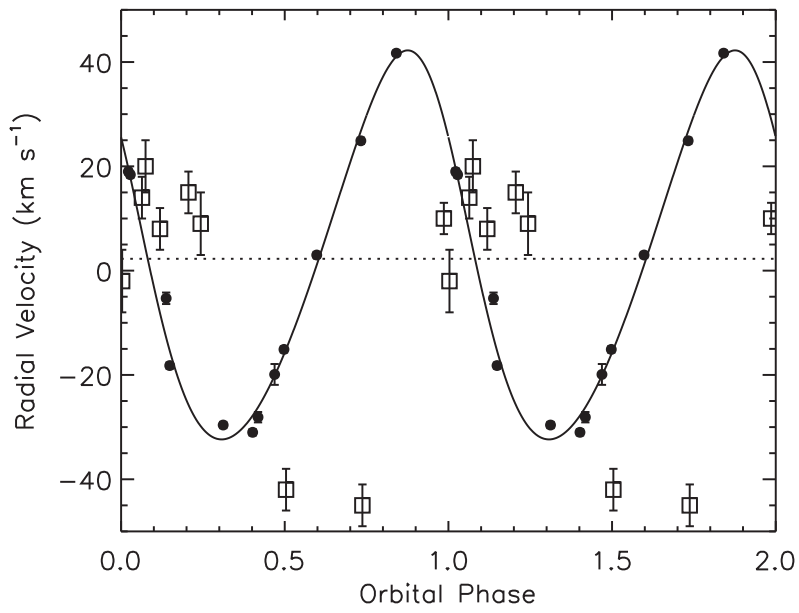

Figure 7. RV curve and orbital solution for the SB1 HDE 305536 at the best-fitting orbital period of $1.88535 \mathrm{~d}$. Solid circles are measurements from our CHIRON spectra. Open squares are data from Levato et al. (1990), shown for comparison but not used to fit the orbital solution. The dotted line marks the systemic velocity.

with an extended arc of $8 \mu \mathrm{m}$ emission indicative of a stellar wind bow shock, which in this case points in the general direction of $\mathrm{Tr}$ 16 (Sexton et al. 2015). HDE 305536 was observed multiple times by Levato et al. (1990), who detected significant RV variations and fit a rough orbit with a period of $2.018 \mathrm{~d}$.

We obtained 12 CHIRON spectra of this system over 2014-2016. The parameters of the best-fitting orbital solution to our data, which has a period of $1.88535 \pm 0.00002 \mathrm{~d}$, are given in Table 4 and the corresponding phase-folded RV curve is shown in Fig. 7. RVFIT was unable to converge on a solution when the RVs from Levato et al. (1990) were included; we overplot those data, folded at our bestfitting CHIRON-based period, for comparison only. Relative to our CHIRON measurements, the Levato et al. (1990) data appear offset by $10-30 \mathrm{~km} \mathrm{~s}^{-1}$ in RV and/or $0.2-0.3$ in phase. As discussed in Section 2.4, many Levato et al. (1990) sources show unexplained $\mathrm{RV}$ offsets relative to later observations. In this case, the apparent phase shift may also suggest the possibility of orbital precession.
The orbit of HDE 305536 is slightly eccentric with $e=0.129 \pm 0.008$. The mass function of the system is $f\left(M_{1}, M_{2}\right)$ $\approx 0.01 \mathrm{M}_{\odot}$. Assuming the mass of the $09.5 \mathrm{~V}$ primary star is $\sim 16 \mathrm{M}_{\odot}$ (Martins et al. 2005), the minimum mass of the secondary star is $\sim 1.4 \mathrm{M}_{\odot}$, equivalent to a mid F-type dwarf (Drilling \& Landolt 2000).

\subsection{Additional spectroscopic binaries}

In addition to HD 93403 and the four spectroscopic binaries described above, six other stars showed significant $\left(P\left(\chi^{2}, v\right)<0.01\right)$ RV variations with amplitudes $>20 \mathrm{~km} \mathrm{~s}^{-1}$. These variations are larger than typical photospheric variability (e.g. Martins et al. 2015), making these stars probable but unconfirmed spectroscopic binaries. We briefly discuss each of these sources below.

ALS 15204: there are no literature RV data for this O7.5 V star (Sota et al. 2014) in $\operatorname{Tr} 14$. We observed it three times with CHIRON in 2014. In spectra taken $16 \mathrm{~d}$ apart, its RV changed by more than $100 \mathrm{~km} \mathrm{~s}^{-1}$. Owing to this star's relative faintness $(V=10.9 \mathrm{mag}$; Hur et al. 2012), we did not pursue follow-up observations in 2015.

ALS 15206: we observed ALS 15206, an O9.2 V star (Sota et al. 2014) in Tr 14, a total of three times with CHIRON in December 2014. Our observations show variability that is significant but low amplitude $\left(\Delta \mathrm{RV} \sim 10 \mathrm{~km} \mathrm{~s}^{-1}\right)$. However, its single-epoch $\mathrm{RV}$ from Huang \& Gies (2006) is $\sim 30 \mathrm{~km} \mathrm{~s}^{-1}$ blueshifted relative to our CHIRON numbers, suggesting a higher degree of variability. ALS 15206 is associated with an extended arc of $8 \mu \mathrm{m}$ emission, likely a stellar wind bow shock, that points north-west towards the centre of $\operatorname{Tr} 14$ (Sexton et al. 2015). It has a local proper motion of $\lesssim 30 \mathrm{~km} \mathrm{~s}^{-1}$, directed to the north-east (Kiminki et al. 2017). As with ALS 15204, we did not pursue follow-up CHIRON observations because this star is relatively faint at visual magnitudes ( $V=10.7 \mathrm{mag}$; Hur et al. 2012)

ALS 15207: this O9 V star (Sota et al. 2014) in Tr 14 was flagged by Levato et al. (1991b) as an SB2; however, García et al. (1998) observed no line doubling nor any significant RV variations. We obtained a total of seven CHIRON spectra of ALS 15207 in 20142015; like García et al. (1998), we found that its He I and He II absorption lines were well fitted with single Gaussian profiles. We measured RVs ranging from -21.5 to $+15.0 \mathrm{~km} \mathrm{~s}^{-1}$ but were unable to constrain the period of the variability.

CPD-59 2554: although this O9.5 IV star (Sota et al. 2014) in Cr 228 was observed by Levato et al. (1990) to be a constant-RV source, our nine CHIRON spectra from 2014 to 2015 show significant variability, with RVs ranging from -22.7 to $+63.4 \mathrm{~km} \mathrm{~s}^{-1}$. We were unable to constrain the period of the variability.

HD 93028: this O9 IV star (Sota et al. 2014) is located about 6 pc south-west of the centre of $\mathrm{Cr} 228$. Levato et al. (1990) identified it as a spectroscopic binary and fit a rough orbit with a period of $51.554 \mathrm{~d}$. We obtained four CHIRON spectra of this source in 2014, which showed significant RV variations at an amplitude just above our $20 \mathrm{~km} \mathrm{~s}^{-1}$ cut-off; a larger amplitude is seen when data from Feast et al. (1957) and Conti et al. (1977) are included. Sota et al. (2014) report that HD 93028 is in a long-period $(\sim 200 \mathrm{~d})$ spectroscopic binary, but do not provide an orbital solution.

$H D E$ 305525: at about $7 \mathrm{pc}$ south-east of $\operatorname{Tr} 16$, this $05.5 \mathrm{~V}$ star (Sota et al. 2014) is part of the distributed population in the South Pillars. Levato et al. (1990) detected significant RV variability in its spectra but were unable to find an orbital solution. Our eight CHIRON spectra from 2014 to 2015 confirm RV variations with an amplitude of more than $130 \mathrm{~km} \mathrm{~s}^{-1}$. Like Levato et al. (1990), we were unable to constrain the period of the variability. 


\section{RESULTS AND DISCUSSION}

\subsection{Distribution of stellar radial velocities}

Combining new spectroscopy with literature data, we have compiled heliocentric RVs for 59 O-type systems in the Carina Nebula, as well as for the LBV $\eta$ Car and the three WNH stars in the region. We present a histogram of these RVs in Fig. 8. The stars are divided into three groups, characterizing the likelihood that the observed $\mathrm{RV}$ s represent their true systemic motions. The first group, shown in light grey in Fig. 8, are sources with relatively reliable RVs. These are spectroscopic binaries with well-constrained orbital solutions, for which we plot the computed systemic velocity, as well as stars with no known RV variability or insignificant or low-amplitude RV variability, for which we plot the weighted mean of the observed RVs. In total, this group, which we will henceforth refer to as the 'well-constrained sources,' consists of $\eta$ Car and 40 O-type stars. The weighted mean RV of the well-constrained sources is $0.6 \mathrm{~km} \mathrm{~s}^{-1}$, with a standard deviation of $9.1 \mathrm{~km} \mathrm{~s}^{-1}$.

The second group of sources, shown in dark grey in Fig. 8, are those that have been identified as spectroscopic binaries but lack orbital solutions. These include stars that were identified as SB1s or SB2s in the multiplicity survey of Chini et al. (2012), stars with binarity flagged elsewhere in the literature, and stars whose CHIRON and/or literature RVs display significant variability with amplitudes $>20 \mathrm{~km} \mathrm{~s}^{-1}$. We refer to this group of 19 O-type systems as the 'unsolved binaries.' For each system, we plot the weighted mean of its measured RVs, but caution that this number may not be representative of its true systemic velocity, especially for systems with few published RVs. For instance, the most notable outlier in Fig. 8 is HD 93130, for which there is only a single reliable published RV (59.9 $\mathrm{km} \mathrm{s}^{-1}$; Conti et al. 1977). However, Chini et al. (2012) classify this source as an SB2, suggesting that its outlying single-epoch RV is the result of its binarity rather than its

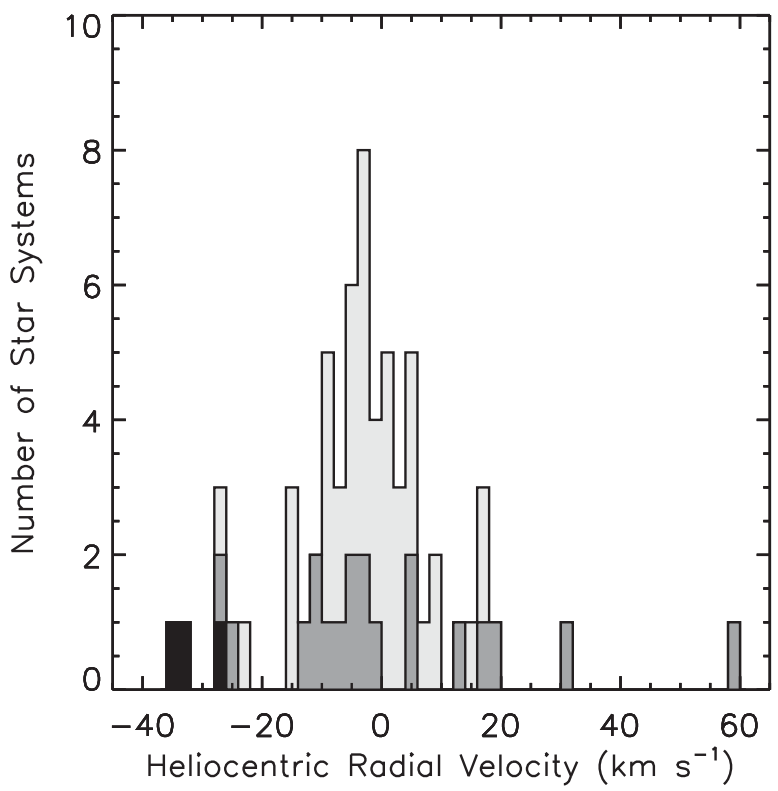

Figure 8. Histogram of the heliocentric RVs of the O-type and evolved massive stars in the Carina Nebula. Well-constrained sources (solved spectroscopic binaries and stars with little to no RV variation) are shown in light grey. Unsolved binaries are shown in dark grey. The three WNH stars, whose reported RVs are affected by strong stellar winds, are indicated in black. relationship with the Carina Nebula. The overall weighted mean RV of the well-constrained sources and the unsolved binaries together remains $0.6 \mathrm{~km} \mathrm{~s}^{-1}$, while the standard deviation of the distribution increases to $13.5 \mathrm{~km} \mathrm{~s}^{-1}$.

The third group of sources, shown in black in Fig. 8, are the WNH stars. As discussed in Section 2.4, the N IV $\lambda 4058$ RVs adopted for these sources are affected by their strong stellar winds. All three fall on the negative end of the Carina Nebula's RV distribution, offset by roughly $-30 \mathrm{~km} \mathrm{~s}^{-1}$ from the mean velocity of the O-type systems. We cannot rule out the possibility that the WNH stars are a distinct kinematic group or that one or more of them is moving at outlying speeds after having been ejected from the region's clusters. However, given that the three stars show a similar RV offset, wind effects are most likely the primary cause.

The overall RV distribution in Fig. 8 is unimodal, consistent with the various clusters and subclusters of the Carina Nebula being part of a single complex at a common distance. We do not see any probable runaway stars. Aside from WR 24 and WR 25, only HD 93130 has an RV that deviates from the region's mean by $\geq 30 \mathrm{~km} \mathrm{~s}^{-1}$, and as described above, HD 93130 is a poorly studied spectroscopic binary and its given RV is unlikely to be its true systemic velocity. However, with RV data, we cannot rule out the presence of runaways with high tangential velocities. Our region of study extends $\sim 20 \mathrm{pc}$ in all directions from $\operatorname{Tr} 16$, meaning a star with a tangential velocity of $30 \mathrm{~km} \mathrm{~s}^{-1}$ would have exited our field in $\sim 600000$ yr. An O-type star ejected by the recent supernova explosion of its companion (see, e.g. Blaauw 1961) might still appear to be within the coordinates of the Carina Nebula, but we see no evidence for this in the RV data.

The standard deviation of the RVs of the well-constrained sources, $9.1 \mathrm{~km} \mathrm{~s}^{-1}$, is an upper limit on the true one-dimensional velocity dispersion of the Carina Nebula, as this group of sources may still contain long-period spectroscopic binaries or other undetected RV variables. Typical OB associations like Scorpius-Centaurus and Perseus OB2 have one-dimensional velocity dispersions of $1-3 \mathrm{~km} \mathrm{~s}^{-1}$ (de Bruijne 1999; Steenbrugge et al. 2003), but these associations are substantially smaller in mass and extent - and slightly older - than the Carina Nebula complex (see Bally 2008; Preibisch \& Mamajek 2008). More directly comparable to the Carina Nebula is the Cygnus OB2 association, which contains $>50$ O-type stars distributed across tens of parsecs (Massey \& Thompson 1991; Wright, Drew \& Mohr-Smith 2015). In both tangential and radial velocities (RVs), the one-dimensional velocity dispersion of Cyg OB2 is $\sim 10 \mathrm{~km} \mathrm{~s}^{-1}$ (Kiminki et al. 2007, 2008; Wright et al. 2016), similar to what we have measured for the O-type stars in the Carina Nebula. This is about a factor of two higher than the velocity dispersions seen in the massive, compact starburst clusters R136 (Hénault-Brunet et al. 2012), NGC 3603 (Rochau et al. 2010), and the Arches Cluster (Clarkson et al. 2012).

\subsection{Variations between clusters}

In Fig. 9, we separate out and compare the RVs of the O-type and evolved massive stars in $\operatorname{Tr} 14$ and $\operatorname{Tr} 16$. We define membership in each cluster as being within a projected 5 arcmin of the cluster centre. This is the observed radius of $\operatorname{Tr} 14$ 's pre-main-sequence population (Ascenso et al. 2007) and a natural break in its O-star distribution. Tr 14 contains six O-type stars with well-constrained RVs and an additional nine unsolved binaries. Tr 16 is larger and less well defined than $\operatorname{Tr} 14$ and is elongated rather than spherical (e.g. Feigelson et al. 2011), and so is poorly defined by a single radius. With a 5 arcmin cut-off, Tr 16 contains 11 sources with 


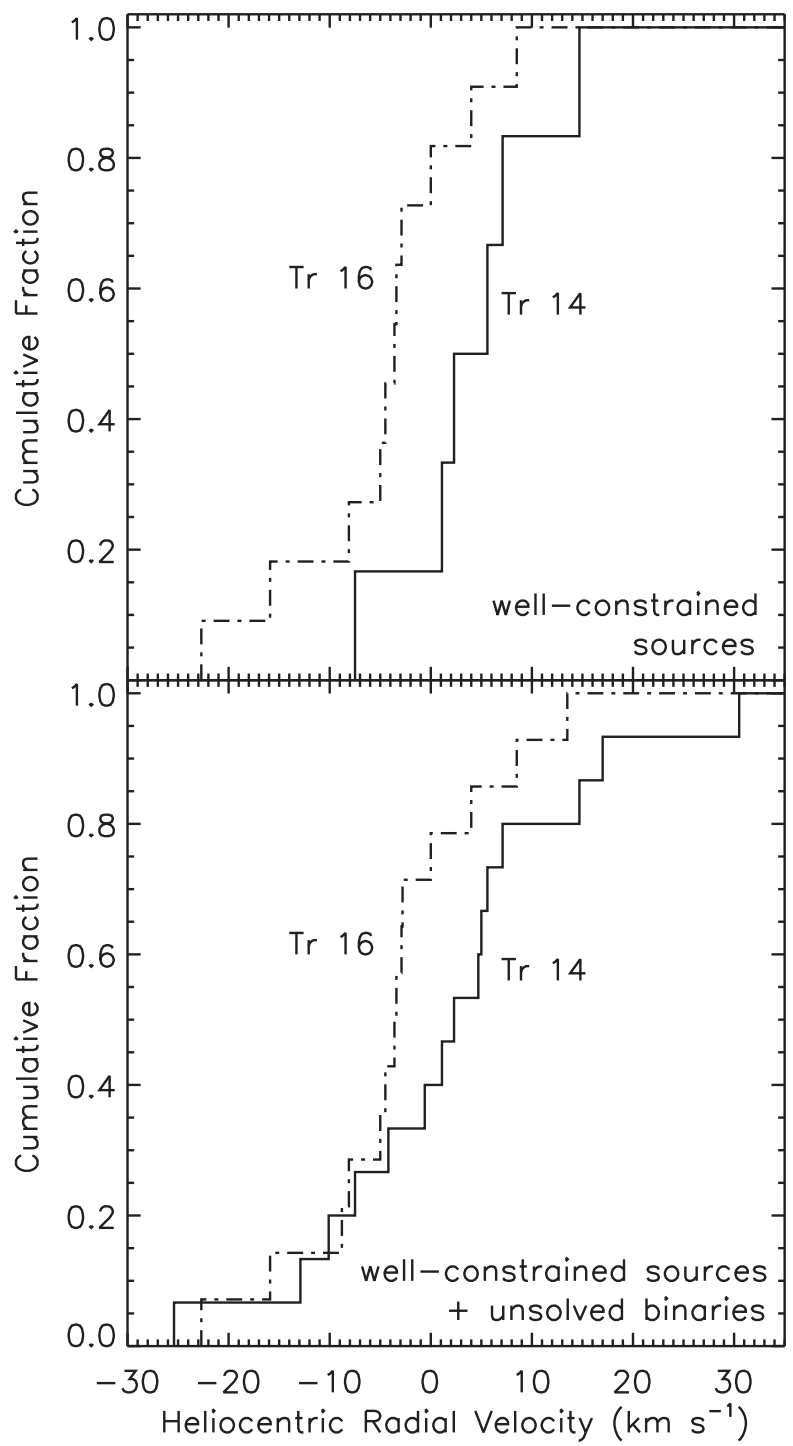

Figure 9. Cumulative histograms of the heliocentric RVs of O-type stars in $\operatorname{Tr} 14$ (solid line) and O-type stars and $\eta$ Car in Tr 16 (dot-dashed line). Both clusters are defined with a radius of $5 \mathrm{arcmin}$. Top: stellar systems with well-constrained RVs (either solved spectroscopic binaries or sources with little to no RV variation). Bottom: as above, but including the mean RVs of unsolved spectroscopic binaries.

well-constrained RVs (including $\eta$ Car), and three unsolved binaries. As we describe below, increasing the radius of Tr 16 slightly increases its velocity dispersion but has little effect on the weighted mean of its RV distribution.

The cumulative histograms in Fig. 9 suggest that the O-type stars in $\operatorname{Tr} 14$ might have slightly more positive RVs, on average, than those in $\operatorname{Tr} 16$. The weighted mean of the well-constrained sources in $\operatorname{Tr} 14$ is $2.3 \pm 7.4 \mathrm{~km} \mathrm{~s}^{-1}$, in agreement with the $2.8 \pm 4.9 \mathrm{~km} \mathrm{~s}^{-1}$ measured by Penny et al. (1993) and the $6.0 \pm 1.4 \mathrm{~km} \mathrm{~s}^{-1}$ found by García et al. (1998). In comparison, the weighted mean of the well-constrained sources within a 5 arcmin radius of $\operatorname{Tr} 16$ is $-3.5 \pm 8.6 \mathrm{~km} \mathrm{~s}^{-1}$. Increasing the radius of $\operatorname{Tr} 16$ to $10 \mathrm{arcmin}$ changes this to $-3.3 \pm 10.4 \mathrm{~km} \mathrm{~s}^{-1}$. To evaluate the significance of the apparent RV difference between clusters, we ran a two-sided Kolmogorov-Smirnov (K-S) test, which evaluates the probability that the two clusters were drawn from the same RV distribution. We estimated the uncertainty on the K-S probability by drawing
$10^{4}$ combinations of each cluster's RVs, randomly permuting each star's RV within a Gaussian distribution with a standard deviation corresponding to that star's observed RV uncertainty. For the wellconstrained sources (the top panel of Fig. 9), the probability that the two clusters come from the same RV distribution is $4_{-3}^{+19}$ percent, meaning the difference in RVs is marginally significant. Including unsolved binaries (as in the bottom panel of Fig. 9) brings the probability of a shared RV distribution to $16_{-13}^{+45}$ per cent, indicating that the difference is not significant. Further study of the kinematics of Tr 14 and Tr 16 are needed to determine if any overall RV difference persists in their lower mass stellar populations. Damiani et al. (2017) found a mean RV of $-5 \mathrm{~km} \mathrm{~s}^{-1}$ for the FGK-type populations of $\operatorname{Tr} 14$ and $\operatorname{Tr} 16$ combined, with no apparent bimodality in their RV distribution, but they did not attempt to separate their stars by cluster.

In Fig. 10, we search for any additional cluster-scale or spatial dependencies by mapping our RVs as a function of position. The circle representing each star is scaled by the magnitude of its mean/systemic RV and colour coded as redshifted (positive RV) or blueshifted (negative RV). The left-hand plot includes only those sources with well-constrained RVs, while the right-hand plot shows both the well-constrained sources and the unsolved binaries. For clarity, we mark only the positions and not the RVs of the three WNH stars.

The apparent velocity difference between $\operatorname{Tr} 14$ and $\operatorname{Tr} 16$ is visible in Fig. 10, in that the former has more red (positive-RV) sources than the latter. Another notable structure is the grouping of O-type stars extending from the nominal centre of $\mathrm{Cr} 228$ up towards $\operatorname{Tr} 16$. These sources have low-magnitude RVs and appear as a sequence of relatively small circles. Their weighted mean $\mathrm{RV}\left(0.3 \mathrm{~km} \mathrm{~s}^{-1}\right)$ is similar to that of the region as a whole, but their velocity dispersion $\left(4.0 \mathrm{~km} \mathrm{~s}^{-1}\right)$ is comparatively small. This relatively low velocity dispersion suggests that this string of massive stars formed in place; we would expect a population that had migrated out from $\operatorname{Tr} 16$ to have a higher velocity dispersion than the cluster rather than vice versa.

\subsection{Comparison to molecular gas}

The molecular cloud complex associated with the Carina Nebula is composed of three main parts (de Graauw et al. 1981; Brooks, Whiteoak \& Storey 1998; Yonekura et al. 2005; Rebolledo et al. 2016). The Northern Cloud partially surrounds Tr 14 and extends to the north-west (de Graauw et al. 1981; Brooks et al. 2003), where it connects to additional molecular material around the Gum 31 H II region (e.g. Rebolledo et al. 2016). The Southern Cloud coincides with the optically dark lane between Tr 16 and the South Pillars (de Graauw et al. 1981). The South Pillars are themselves composed of molecular gas, shaped by feedback from Carina's OB stars (Rathborne et al. 2004; Yonekura et al. 2005; Rebolledo et al. 2016). Within Tr 16, there are only scattered molecular globules, thought to be the remnants of the gas from which that cluster formed (Cox \& Bronfman 1995; Brooks et al. 2000).

Rebolledo et al. (2016) observed the Carina Nebula in ${ }^{12} \mathrm{CO}(1-0)$ emission as part of the Mopra Southern Galactic Plane CO Survey (Burton et al. 2013). We use their data to compare the kinematics of Carina's molecular gas to the RVs of its O-type and evolved massive stars. For Figs 11 and 12, we collapse their three-dimensional data cubes along Galactic latitude and longitude, respectively, to present two-dimensional position-velocity diagrams of the dense gas in Carina. We convert the gas velocities from the local standard of rest 


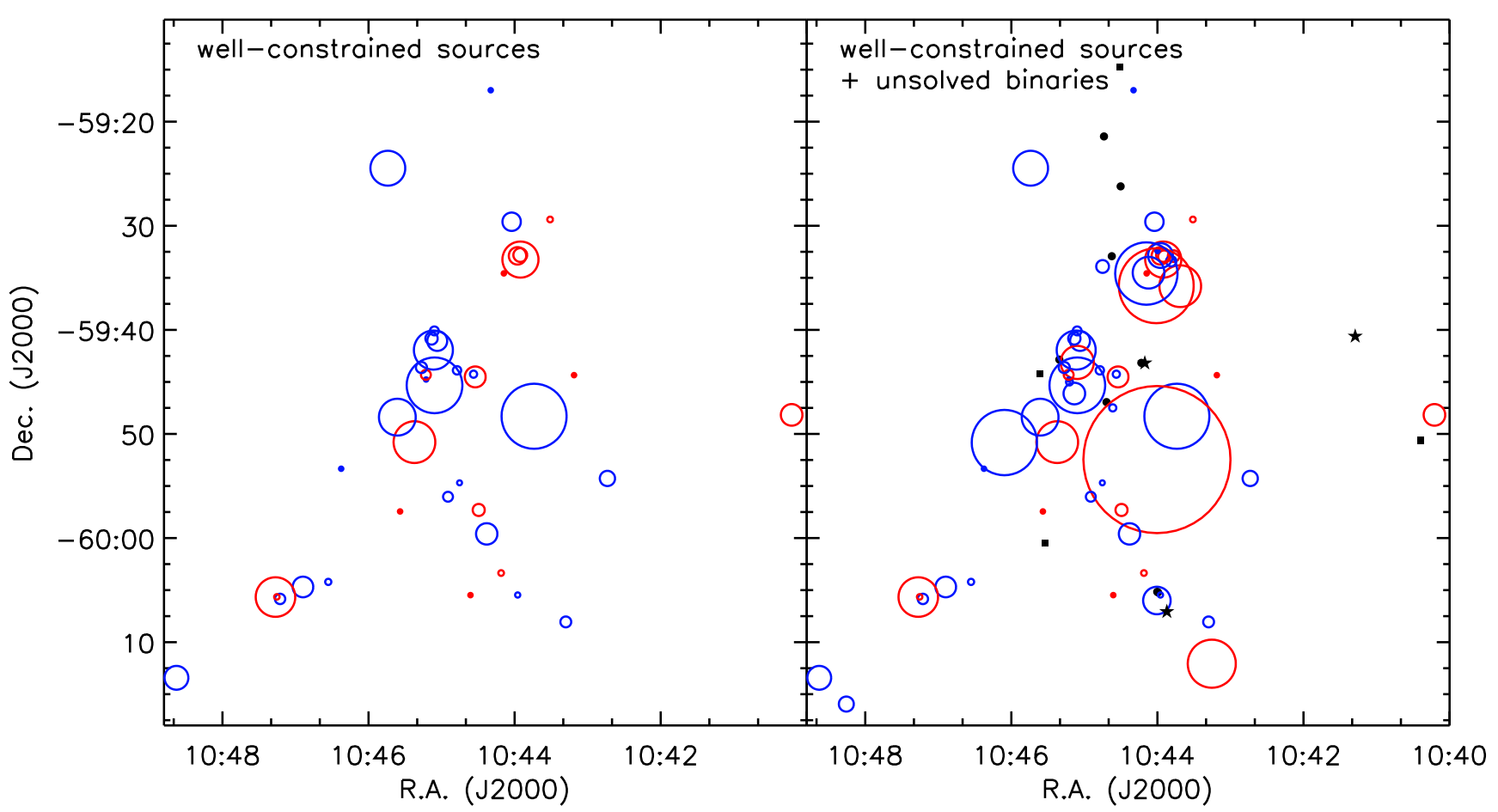

Figure 10. Maps of the heliocentric RVs of O-type and evolved massive stars in the Carina Nebula. Left: stars (including $\eta$ Car) with well-constrained RVs as defined in the text. Blue circles indicate negative RVs and red circles indicate positive RVs; circle size scales with the magnitude of the velocity. Right: as left, but including known spectroscopic binaries that lack orbital solutions. The filled black circles and squares show the positions of O-type stars from v3.1 of the GOSC and from Alexander et al. (2016), respectively, that lack RV data. The positions of the three WNH stars are shown by black filled stars.

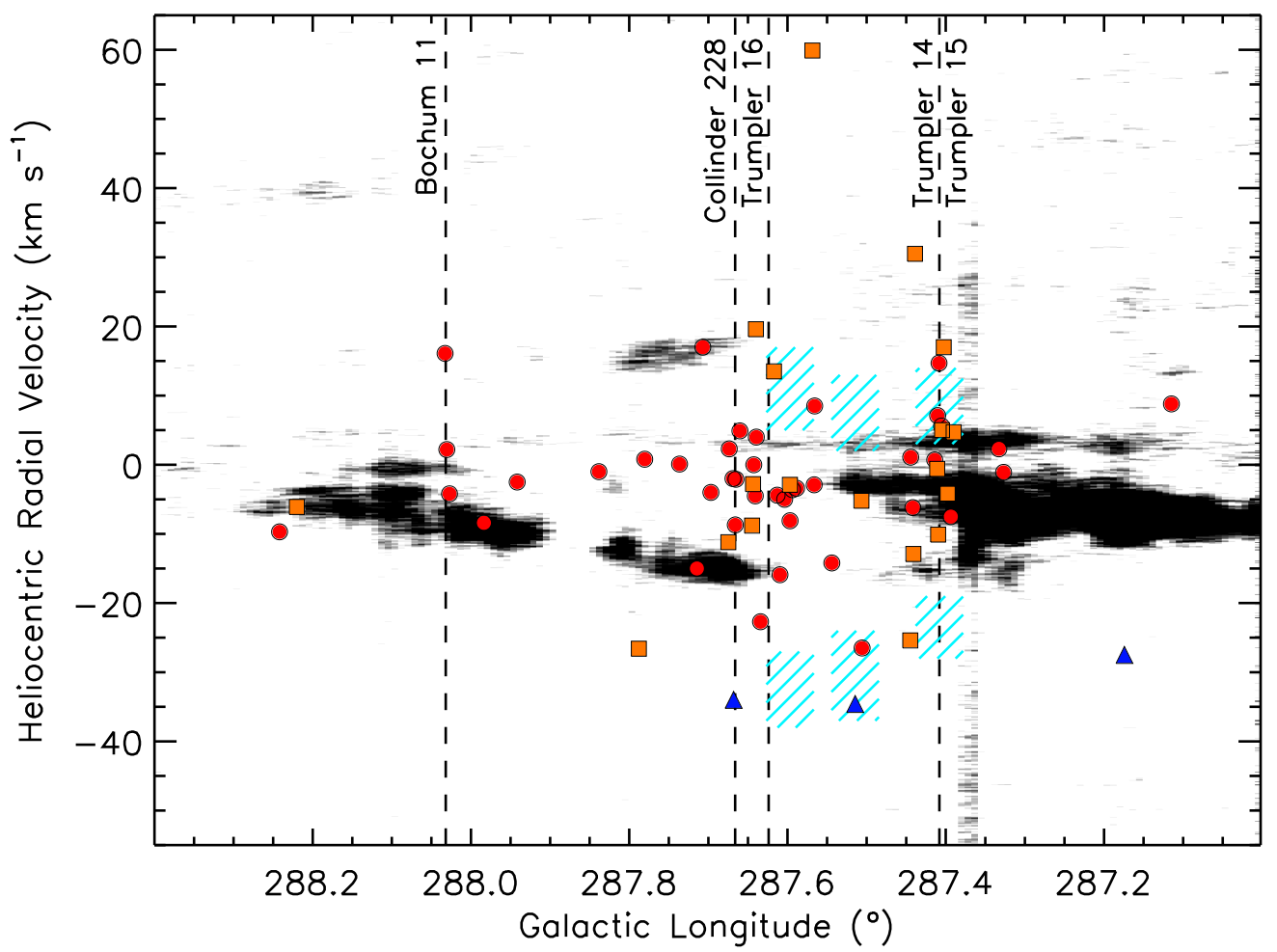

Figure 11. Position-velocity diagram of ${ }^{12} \mathrm{CO}(1-0)$ emission (grey scale) from Rebolledo et al. (2016, converted to a heliocentric frame) compared to O-type and massive evolved stars. Red circles are systems (including $\eta$ Car) with well-constrained RVs, orange squares are known or suspected spectroscopic binaries that lack orbital solutions, and blue triangles are WNH stars. The hatched cyan regions show the RVs of the approaching and receding components of emission from ionized gas (Damiani et al. 2016) centred on the positions of $\eta$ Car, WR 25, and $\operatorname{Tr} 14$. 


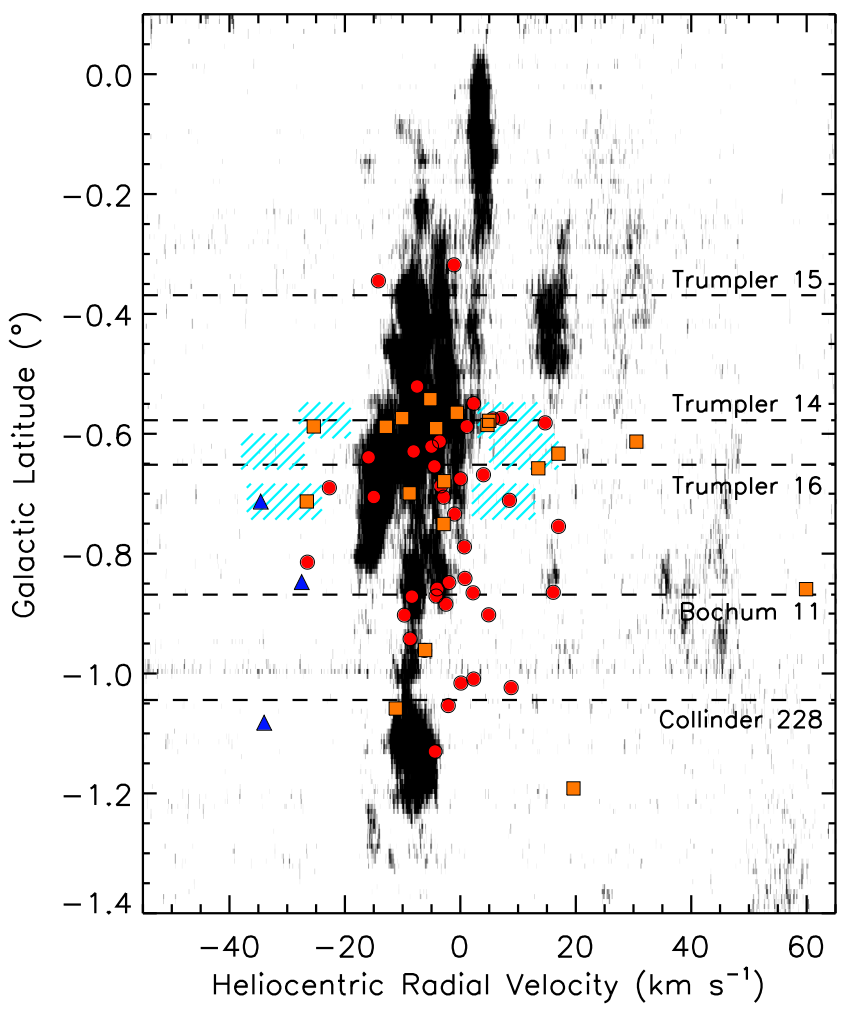

Figure 12. Similar to Fig. 11, but showing the heliocentric RVs of ${ }^{12} \mathrm{CO}$, ionized gas, and stars as a function of Galactic latitude.

to a heliocentric frame. At the coordinates of the Carina Nebula, $R V_{\text {helio }} \approx R V_{\mathrm{LSR}}+11.6 \mathrm{~km} \mathrm{~s}^{-1}$. All $\mathrm{RVs}$ in the following discussion are heliocentric unless otherwise noted.

Fig. 11 plots the RVs of the Carina Nebula's O-type stars, evolved massive stars, and ${ }^{12} \mathrm{CO}(1-0)$ emission as a function of Galactic longitude $l$. The ${ }^{12} \mathrm{CO}$ fluxes from Rebolledo et al. (2016) are summed across Galactic latitudes $-1.4 \leq b \leq 0.1$. As in Fig. 8, the stars are divided into three groups: stars (including $\eta$ Car) with well-constrained systemic velocities, unsolved binaries, and $\mathrm{WNH}$ stars. We also indicate the approximate heliocentric velocities of the redshifted and blueshifted components of ionized gas emission lines observed by Damiani et al. (2016) for shells centred on the locations of $\eta$ Car, WR 25, and Tr 14. In Fig. 12, we present the same data, but plotted as a function of Galactic latitude $b$. The ${ }^{12} \mathrm{CO}$ fluxes are summed across Galactic longitudes $287.0 \leq l \leq 288^{\circ} .4$.

The three components of Carina's molecular cloud complex separate cleanly when plotted against Galactic longitude in Fig. 11: the Northern Cloud at $l \approx 287.0-287.5$ with heliocentric RV $\approx(-10)$ $0 \mathrm{~km} \mathrm{~s}^{-1}$; the Southern Cloud at $l \approx 287.6-287.8$ with heliocentric $\mathrm{RV} \approx(-15)-(-10) \mathrm{km} \mathrm{s}^{-1}$; and the South Pillars at $l \approx 287.9$ 288.2 with heliocentric $\mathrm{RV} \approx(-10)-(-5) \mathrm{km} \mathrm{s}^{-1}$ and a smaller part at $\sim 0 \mathrm{~km} \mathrm{~s}^{-1}$. The separation is less notable in Fig. 12, as the sourthern part of the Northern Cloud overlaps with the Southern Cloud in Galactic latitude, but the RV offset in the Southern Cloud is still apparent. The molecular gas at $l \approx 287.7-287.8, b \approx-0.4$, with heliocentric $\mathrm{RV} \sim+20 \mathrm{~km} \mathrm{~s}^{-1}$, is thought to be associated with the far side of the Carina arm, at a greater distance than the Carina Nebula (Damiani et al. 2016).

Figs 11 and 12 further emphasize that the RVs of the O-type stars in the Carina Nebula are not spatially dependent, as there is no trend in O-star RVs with Galactic longitude or latitude. Rebolledo et al. (2016) applied the four-arm Milky Way model of Vallée (2014) to the rotation curve of McClure-Griffiths \& Dickey (2007) and calculated the expected RV for objects at various distances along the tangent of the Carina spiral arm. For sources on the near side of the arm, at $\sim 2 \mathrm{kpc}$, the expected local standard of rest RV is approximately $-10 \mathrm{~km} \mathrm{~s}^{-1}$, which corresponds to a heliocentric RV of $\approx+2 \mathrm{~km} \mathrm{~s}^{-1}$, very close to the observed mean RV of the O-type stars. Objects at greater distances from the Sun would be expected to have more positive RVs (see Rebolledo et al. 2016). Our RV results thus favour the OB clusters and groups in the Carina Nebula being at a common distance of approximately $2 \mathrm{kpc}$.

\subsection{1 $\operatorname{Tr} 14, \operatorname{Tr} 15$, and the Northern Cloud}

There is a general consensus that the relatively compact $\operatorname{Tr} 14$ is the youngest of the Carina Nebula's Trumpler clusters, at just 1-2 Myr old (Walborn 1973, 1982a,b, 1995; Morrell et al. 1988; Vazquez et al. 1996; Smith \& Brooks 2008; Rochau et al. 2011). The case for its youth is strengthened by its close spatial association with the Northern Cloud, which wraps around the west side of the cluster (de Graauw et al. 1981; Brooks et al. 2003; Tapia et al. 2003). Bright radio emission arises from multiple ionization fronts where radiation from $\operatorname{Tr} 14$ impacts dense clumps in the Northern Cloud (Whiteoak 1994; Brooks, Storey \& Whiteoak 2001). It is clear from Figs 11 and 12 that the O-type stars in Tr 14 are also kinematically associated with the Northern Cloud, further strengthening the picture of $\operatorname{Tr} 14$ as a young cluster that has not yet dispersed its natal molecular gas.

While $\operatorname{Tr} 15$ has sometimes been considered an unrelated foreground or background cluster (Thé \& Vleeming 1971; Walborn 1973), its extended X-ray stellar population indicates a connection to $\operatorname{Tr} 14$ (Feigelson et al. 2011; Wang et al. 2011). We have RV data for only two O-type stars around Tr 15, HD 93403 and HD 93190, which appear at $b \sim-0.35$ in Fig. 12. Their RVs are consistent with those of the rest of the O-type stars in the Carina Nebula and span the RV range of the Northern Cloud. Both are 4-5 pc outside the core of $\operatorname{Tr} 15$, and neither is associated with any group or subcluster (see Feigelson et al. 2011). Tr 15 is likely several Myr older than Carina's other Trumpler clusters (Carraro 2002; Tapia et al. 2003; Wang et al. 2011), and it seems likely that these two stars drifted out from $\operatorname{Tr} 15$ over the course of their lifetimes.

Off the southern edge of the Northern Cloud, on the far west side of the Carina Nebula (see Fig. 1), there are several O-type and evolved massive stars, notably WR 22 and HD 92607. There is a young $(<1 \mathrm{Myr})$ pre-main-sequence population in this region, although it does not show any clustering around the massive stars (Kumar et al. 2014). WR 22 is a particularly puzzling system: it is a very massive binary ( 55-M $\odot$ primary; Schweickhardt et al. 1999) approximately $21 \operatorname{arcmin}(15 \mathrm{pc})$ from $\operatorname{Tr} 14$, without any subcluster or group of its own. Its N IV $\lambda 4058 \mathrm{RV}$ is somewhat less negative than the RVs of WR 24 and WR 25, but it is unclear whether this represents a true difference in their systemic motions or whether it is the result of uncharacterized differences in their stellar winds.

The SB2 HD 92607 (see Section 3.1), evident on the far righthand side of Fig. 11, is less extreme in mass but still somewhat puzzling in origin. Its systemic $\mathrm{RV}\left(8.8 \mathrm{~km} \mathrm{~s}^{-1}\right)$ is relatively positive for an O-type system in the Carina Nebula, although still within one standard deviation of the mean. And despite its proximity to the Northern Cloud, it lacks a kinematic association with the molecular gas - perhaps a hint that it did not form in its currently observed location. HD 92607 is associated with a candidate bow shock in the form of a resolved $24 \mu \mathrm{m}$ arc pointing to the south-east (Sexton et al. 2015), which might suggest an origin outside the Carina Nebula. 
However, in the active environment of this giant $\mathrm{H}$ II region, bow shocks are not clear indicators of stellar motion (Kiminki et al. 2017). Its spectroscopic parallax (using data from Martins et al. 2005; Gagné et al. 2011; Povich et al. 2011b) and the inferred distance from its Gaia DR1 geometric parallax (Astraatmadja \& Bailer-Jones 2016) are both consistent with HD 92607 being part of the Carina Nebula at a distance of around $2 \mathrm{kpc}$.

\subsection{2 $\operatorname{Tr} 16$ and the Southern Cloud}

Unlike the Northern Cloud, which has a close kinematic association with the $\operatorname{Tr} 14$ cluster, the Southern Cloud is offset by $10-15 \mathrm{~km} \mathrm{~s}^{-1}$ in RV from its neighbour Tr 16. The Southern Cloud has the most negative RVs of the molecular gas in Carina, being $5-10 \mathrm{~km} \mathrm{~s}^{-1}$ blueshifted relative to both the Northern Cloud and the molecular gas in the South Pillars. It coincides with a prominent, optically dark dust lane, indicating that it lies in front of the south-eastern edge of Tr 16 (Dickel 1974; de Graauw et al. 1981; Brooks et al. 1998). Inside Tr 16 itself, there are only small clumps of molecular gas (not visible in the Rebolledo et al. 2016 data), and these have RVs more consistent with those of the O-type stars in the cluster (Cox \& Bronfman 1995; Brooks et al. 2000).

One of the key arguments in favour of $\operatorname{Tr} 16$ being $1-2 \mathrm{Myr}$ older than Tr 14 (e.g. de Graauw et al. 1981; Walborn 1995) is that the latter (in addition to being more compact) is still partially enclosed by its natal molecular cloud, while the former appears to have disrupted the gas from which it formed. The distribution of gas RVs in Fig. 11 suggests that the Northern Cloud, Southern Cloud, and Southern Pillars were originally part of a single continuous molecular cloud, with the massive stars in Tr 16 having since blown out the central part of that cloud. The Northern Cloud, currently being eroded by $\operatorname{Tr} 14$, has not been accelerated along our line of sight, while the molecular gas in the South Pillars has undergone some acceleration at its closest approach to $\operatorname{Tr} 16$.

The acceleration of the Southern Cloud by the O-type stars in $\mathrm{Tr}$ 16 is readily explained by the rocket effect (Oort \& Spitzer 1955; Bally \& Scoville 1980; Bertoldi \& McKee 1990). As the neutral gas facing $\operatorname{Tr} 16$ is ionized by the strong ultraviolet radiation of the cluster (Smith 2006), it flows away from the surface of the molecular cloud at roughly its sound speed. The recoil force on the molecular cloud causes it to accelerate away from the ionizing source at a rate proportional to the rate at which it loses mass through ionization. In the simplest scenario, assuming the cloud is initially at rest relative to the ionizing source, the cloud mass and velocity are connected through (Spitzer 1978):

$M_{\mathrm{c}}=M_{0} \mathrm{e}^{-v_{\mathrm{c}} / V_{\text {ion }}}$,

where $M_{0}$ and $M_{\mathrm{c}}$ are the initial and current masses of the molecular cloud, respectively, $v_{\mathrm{c}}$ is the current velocity of the cloud, and $V_{\text {ion }}$ is the velocity with which the newly ionized material flows away from the cloud. For the molecular cloud to have been accelerated to $\sim 10 \mathrm{~km} \mathrm{~s}^{-1}$, roughly the speed of sound in ionized gas, the cloud's mass must have decreased by $\sim 60$ percent. The current mass of the Southern Cloud is $\sim 5 \times 10^{4} \mathrm{M}_{\odot}$ (Rebolledo et al. 2016). Accounting only for mass loss through ionization, its initial mass would have been on the order of $1.3 \times 10^{5} \mathrm{M}_{\odot}$, comparable to the current mass of the Northern Cloud (Yonekura et al. 2005; Rebolledo et al. 2016).

Noteworthy among the O-type stars in $\operatorname{Tr} 16$ is V662 Car at $l=287.7, b=-0.7$, an eclipsing spectroscopic binary with a systemic velocity of $-15 \pm 2 \mathrm{~km} \mathrm{~s}^{-1}$ (Niemela et al. 2006). V662 Car is the only O-type star to coincide with the Southern Cloud in three-dimensional position-velocity space, and its relatively high visual extinction (Smith 1987; Povich et al. 2011a) suggests that it is behind or within the molecular gas. The primary star's spectrum has unusually strong He II $\lambda 4686$ (Niemela et al. 2006), leading it to be assigned to the luminosity class Vz (Sota et al. 2014), which is associated with very young O-type stars close to the zeroage main sequence (Walborn 2009; Sabín-Sanjulián et al. 2014; Walborn et al. 2014; Arias et al. 2016). Roughly a dozen of the O-type stars in the Carina Nebula are of class Vz (Sota et al. 2014), with the highest fraction relative to non-z O dwarfs found in $\operatorname{Tr} 14$ (Arias et al. 2016). In addition, Niemela et al. (2006) found that both components of V662 Car have smaller radii and luminosities than expected for their spectral types, another indicator of youth. The combination of V662 Car's young age, its deviation from the mean $\mathrm{RV}$ of the O-type stars in Tr 16, and its kinematic association with the Southern Cloud suggest that it was formed separately from and more recently than the body of $\operatorname{Tr} 16$. We propose that its formation may have been triggered by the feedback-induced acceleration of the Southern Cloud.

\subsubsection{Cr 228, Bo 11, and the molecular gas in the South Pillars}

The relationship between the O-type stars in Bo 11 and $\mathrm{Cr} 228$ and the molecular gas in the South Pillars is difficult to interpret, because the gas detected in ${ }^{12} \mathrm{CO}$ is spatially separate from the $\mathrm{O}$ type stars. Most of the ${ }^{12} \mathrm{CO}(1-0)$ emission in the South Pillars comes from the so-called Giant Pillar (Smith et al. 2000; Yonekura et al. 2005; Smith et al. 2010b), a dusty structure that points towards $\operatorname{Tr} 16$ from the southernmost part of the region, lying roughly halfway between Bo 11 and the nominal centre of $\mathrm{Cr} 228$. The Giant Pillar is the site of current star formation (Smith et al. 2010b; Gaczkowski et al. 2013), but is $\geq 4$ pc from any O-type stars. Its gas has heliocentric RVs of $(-10)-(-5) \mathrm{km} \mathrm{s}^{-1}$, similar to the Northern Cloud. Another dusty structure, whose ionization fronts also face $\operatorname{Tr} 16$, is spatially coincident with Bo 11 . Some ${ }^{12} \mathrm{CO}$ (1-0) emission, too faint to appear in Figs 11 and 12, is detected in this region (Rebolledo et al. 2016), but it has a heliocentric RV of $\gtrsim 30 \mathrm{~km} \mathrm{~s}^{-1}$ and likely belongs to the far side of the Carina spiral arm.

Most of the O-type stars in Bo 11 have RVs similar to the rest of the O-type stars in the Carina Nebula and comparable to the gas in the South Pillars. The exception is HDE 305612, which, with a measured mean RV of $16.1 \mathrm{~km} \mathrm{~s}^{-1}$, appears to be a notable outlier. However, HDE 305612 shows significant RV variation over three epochs of CHIRON data, although it was not flagged as an unsolved binary because the amplitude of that variation is $<20 \mathrm{~km} \mathrm{~s}^{-1}$.

The O-star population of $\mathrm{Cr} 228$ also has similar RVs to the rest of the region; in Fig. 11, it is indistinguishable from the O-star population of $\operatorname{Tr} 16$. None of the many smaller dust pillars around Cr 228 (Smith et al. 2010b) are detected in ${ }^{12} \mathrm{CO}(1-0)$ emission (Rebolledo et al. 2016), ruling out a direct comparison between the RVs of stars and gas in this part of the Carina Nebula. As discussed in Section 4.2, the O-type stars in and around $\mathrm{Cr} 228$ have a lower velocity dispersion than the region as a whole, suggesting that they were not scattered out of $\operatorname{Tr} 16$.

In the far south-east corner of the Carina Nebula, approximately $12.5 \operatorname{arcmin}(\sim 8 \mathrm{pc})$ from Bo 11, are HD 93843 and HDE 305619. These two stars are clearly visible on the left-hand side of Fig. 11 as the two O-type systems with the highest Galactic longitudes. They are typically treated as members of the Carina Nebula complex (e.g. Gagné et al. 2011), although there are no known gas structures or 
lower mass stellar populations connecting them to the rest of the South Pillars (e.g. Smith et al. 2010b). The spectroscopic parallax of HDE 305619 (using data from Kharchenko 2001; Martins et al. 2005; Kharchenko \& Roeser 2009; Gagné et al. 2011; Sota et al. 2014) suggests that it might be in the background at a distance of $>3 \mathrm{kpc}$, but inferred distances based on Gaia DR1 (Astraatmadja \& Bailer-Jones 2016) place both it and HD 93843 at $\sim 2.4 \mathrm{kpc}$. Their observed RVs are also consistent with being part of the Carina Nebula complex, although HDE 305619 shows significant lowamplitude variability in our CHIRON data and was classified as an SB1 by Chini et al. (2012).

\subsection{Comparison to ionized gas}

The ionized gas in the Carina Nebula is globally expanding at $\pm 15-20 \mathrm{~km} \mathrm{~s}^{-1}$, as seen in radio recombination lines (Gardner et al. 1970; Huchtmeier \& Day 1975; Azcarate, Cersosimo \& Colomb 1981; Brooks, Storey \& Whiteoak 2001) and optical line emission (Deharveng \& Maucherat 1975; Walborn \& Hesser 1975; Walsh 1984; Smith, Bally \& Brooks 2004). This expansion is driven by feedback from Carina's O-type and evolved massive stars (Smith \& Brooks 2007). It was most recently mapped by Damiani et al. (2016), who observed more than 650 optical sightlines across $\operatorname{Tr} 14$ and $\operatorname{Tr}$ 16. They identified three non-spherical expanding shells, roughly centred on the positions of $\eta \mathrm{Car}$, WR 25, and Tr 14. We represent these shells in Figs 11 and 12 with hatched regions showing the range of observed approaching and receding RVs for each shell. Damiani et al. (2016) estimate that the overall expansion is centred around an RV of $-12.5 \mathrm{~km} \mathrm{~s}^{-1}$. Their $\operatorname{Tr} 14$ shell is centred around a slightly less negative $-8 \mathrm{~km} \mathrm{~s}^{-1}$. These values agree with prior results: Walborn (1973) and Walborn et al. (2002b, 2007) place the kinematic centre of the expansion at $-14 \mathrm{~km} \mathrm{~s}^{-1}$, and radio data (Gardner et al. 1970; Huchtmeier \& Day 1975; Azcarate et al. 1981) consistently centres the expansion at a heliocentric RV of $-9 \mathrm{~km} \mathrm{~s}^{-1}$.

However, the RV distribution of Carina's O-type and evolved massive stars is not aligned with the expansion of the ionized gas. As described in Section 4.1, the weighted mean RV of the wellconstrained O-type stars is $0.6 \pm 9.1 \mathrm{~km} \mathrm{~s}^{-1}$, putting the kinematic centre of the ionized gas expansion roughly one standard deviation blueward of the mean O-star RV. Figs 11 and 12 show that the receding/redshifted components of the gas overlap in RV space with the positive-RV tail of the O-star distribution, while the approaching/blueshifted components of the gas have more negative RVs than nearly all of the O-type stars. The N IV $\lambda 4058 \mathrm{RV}$ s of the WNH stars, including WR 25, agree with the RVs of the approaching gas, but as previously discussed, this is due to wind effects in the WNH spectra and not due to a physical association.

The most likely explanation for the kinematic asymmetry between the gas and the O-type stars is that the receding/redshifted ionized gas is bounded by dense, neutral material behind the Carina Nebula, while the approaching/blueshifted ionized gas moves freely along our line of sight. In addition, the approaching gas around $\mathrm{Tr}$ 16 may be partially composed of a photoevaporative flow off the Southern Cloud, which has already been accelerated towards us by feedback from Tr 16 (see Section 4.3.2). Damiani et al. (2016) noted a number of smaller scale kinematic asymmetries in the ionized gas shells, confirming that the expansion of the $\mathrm{H}$ II region is non-spherical and is affected by density variations in the surrounding medium. We consequently caution against using the kinematic centre of the ionized gas to define the systemic RV of the Carina Nebula.

\section{CONCLUSIONS}

We have conducted an RV survey of the O-type and evolved massive stars in the Carina Nebula. We obtained multi-epoch echelle spectroscopy for 31 O-type stars, and compiled published RVs for an additional 32 systems including three WNH stars and the LBV $\eta$ Car. With these data, we find the first spectroscopic orbital solutions for the near-twin system HD 92607 and the eclipsing binary HDE 303312 and provide updated orbital solutions for HD 93576 and HDE 305536. Our further results are summarized as follows:

(1) Of the 63 O-type and evolved massive star systems with RV data, 41 have well-constrained systemic velocities. These wellconstrained sources have a weighted mean RV of $0.6 \mathrm{~km} \mathrm{~s}^{-1}$, comparable to prior results for the $\operatorname{Tr} 14$ cluster and to the expected radial motion for sources at a distance of $\sim 2 \mathrm{kpc}$ along the Carina spiral arm.

(2) The standard deviation of the RVs of the well-constrained sources is $9.1 \mathrm{~km} \mathrm{~s}^{-1}$ and provides an upper limit to the onedimensional velocity dispersion of the region. This value is high compared to the velocity dispersions of typical, less massive $\mathrm{OB}$ associations and is roughly twice that of massive bound starburst clusters. However, it is similar to the velocity dispersion of the large, unbound $\mathrm{Cyg}$ OB2 association, and is not unexpected for a region with the content and substructure of the Carina Nebula.

(3) The overall O-star RV distribution is unimodal, favouring a common distance to the various clusters of the Carina Nebula.

(4) There is a possible but marginally significant difference between the RV distributions of the $\operatorname{Tr} 16$ and $\operatorname{Tr} 14$ clusters, with the latter's O-star RVs $\sim 5 \mathrm{~km} \mathrm{~s}^{-1}$ more positive, on average, than the former's. Kinematic study of the intermediate-mass populations in these clusters is needed to confirm the offset.

(5) We do not detect any line-of-sight runaway O-type stars, nor do we see evidence that the distributed O-type population migrated out from $\operatorname{Tr} 14$ and $\operatorname{Tr} 16$. On the contrary, the O-type stars in $\mathrm{Cr}$ 228 and the South Pillars have a low velocity dispersion compared to the region as a whole.

(6) The Tr 14 cluster is kinematically associated with the molecular gas of the Northern Cloud, consistent with its young age.

(7) Feedback from Tr 16 has accelerated the molecular gas of the Southern Cloud towards us, relative to the stellar population, by $10-15 \mathrm{~km} \mathrm{~s}^{-1}$. V662 Car, an O-type star on the outskirts of Tr 16 , may belong to a younger generation of stars triggered by this feedback.

(8) The approaching components of the ionized gas around $\mathrm{Tr}$ 14 and $\operatorname{Tr} 16$ show higher velocities, relative to the O-type stars, than the receding components. This kinematic asymmetry indicates that the expansion of the $\mathrm{H}$ II region is not spherical and is likely impacted by the distribution of dense neutral gas.

Our observations set the stage for the analysis of future Gaia DRs, which will add further kinematic dimensions to our understanding of the Carina Nebula. This region continues to be a laboratory for the study of massive star formation and the interplay between stellar feedback and the interstellar medium.

\section{ACKNOWLEDGEMENTS}

We would like to thank the operators of the CTIO 1.5-m telescope for executing our CHIRON observations, and SMARTS Data/Queue Manager Emily MacPherson for queue scheduling. We also thank Maxwell Moe for his guidance in using NIGHTFALL to fit the orbit of HDE 303312, and we thank the anonymous referee for the time spent reviewing this manuscript. This work is based on observations 
at Cerro Tololo Inter-American Observatory, National Optical Astronomy Observatory (NOAO Prop. IDs: 2014B-0235 and 2015B0141; PI: M. Kiminki), which is operated by the Association of Universities for Research in Astronomy (AURA) under a cooperative agreement with the National Science Foundation. This paper also makes use of data obtained using the Mopra radio telescope, a part of the Australia Telescope National Facility which is funded by the Commonwealth of Australia for operation as a National Facility managed by CSIRO.

Finally, we note that while editing the final revision of this manuscript, we learned of the passing of Nolan R. Walborn. His contributions towards our understanding of massive O-type stars in general have been tremendous, and particularly so for the massive stars in the Carina Nebula studied in this paper.

\section{REFERENCES}

Albacete Colombo J. F., Morrell N. I., Niemela V. S., Corcoran M. F., 2001, MNRAS, 326, 78

Albacete Colombo J. F., Morrell N. I., Rauw G., Corcoran M. F., Niemela V. S., Sana H., 2002, MNRAS, 336, 1099

Alexander M. J., Hanes R. J., Povich M. S., McSwain M. V., 2016, AJ, 152, 190

Allen D. A., 1979, MNRAS, 189, 1P

Allen D. A., Hillier D. J., 1993, PASA, 10, 338

Almeida L. A. et al., 2017, A\&A, 598, A84

Arias J. I. et al., 2016, AJ, 152, 31

Ascenso J., Alves J., Vicente S., Lago M. T. V. T., 2007, A\&A, 476, 199

Astraatmadja T. L., Bailer-Jones C. A. L., 2016, ApJ, 833, 119

Azcarate I. N., Cersosimo J. C., Colomb F. R., 1981, Rev. Mex. Astron. Astrofis., 6, 269

Bally J., 2008, in Reipurth B., ed., Handbook of Star Forming Regions, Vol. I. Astron. Soc. Pac., San Francisco, CA, p. 459

Bally J., Scoville N. Z., 1980, ApJ, 239, 121

Barbá R. H., Gamen R., Arias J. I., Morrell N., Maíz Apellániz J., Alfaro E., Walborn N., Sota A., 2010, in Rivinius T., Curé M., eds, Revista Mexicana de Astronomia y Astrofisica Conference Series Vol. 38, The Interferometric View on Hot Stars. Universidad Nacional Autónoma de México, Mexico, p. 30

Bertoldi F., McKee C. F., 1990, ApJ, 354, 529

Blaauw A., 1961, Bull. Astron. Inst. Netherlands, 15, 265

Bohannan B., Garmany C. D., 1978, ApJ, 223, 908

Brooks K. J., Whiteoak J. B., Storey J. W. V., 1998, PASA, 15, 202

Brooks K. J., Burton M. G., Rathborne J. M., Ashley M. C. B., Storey J. W. V., 2000, MNRAS, 319, 95

Brooks K. J., Storey J. W. V., Whiteoak J. B., 2001, MNRAS, 327, 46

Brooks K. J., Cox P., Schneider N., Storey J. W. V., Poglitsch A., Geis N., Bronfman L., 2003, A\&A, 412, 751

Burton M. G. et al., 2013, PASA, 30, e044

Carraro G., 2002, MNRAS, 331, 785

Carraro G., Patat F., 2001, A\&A, 379, 136

Carraro G., Romaniello M., Ventura P., Patat F., 2004, A\&A, 418, 525

Chini R., Hoffmeister V. H., Nasseri A., Stahl O., Zinnecker H., 2012, MNRAS, 424, 1925

Clark P. C., Bonnell I. A., Zinnecker H., Bate M. R., 2005, MNRAS, 359, 809

Clarkson W. I., Ghez A. M., Morris M. R., Lu J. R., Stolte A., McCrady N., Do T., Yelda S., 2012, ApJ, 751, 132

Coelho P. R. T., 2014, MNRAS, 440, 1027

Collado A., Gamen R., Barbá R. H., 2013, A\&A, 552, A22

Collado A., Gamen R., Barbá R. H., Morrell N., 2015, A\&A, 581, A49

Conti P. S., Leep E. M., Lorre J. J., 1977, ApJ, 214, 759

Conti P. S., Niemela V. S., Walborn N. R., 1979, ApJ, 228, 206

Cox P., Bronfman L., 1995, A\&A, 299, 583

Crowther P. A., 2007, ARA\&A, 45, 177

Cudworth K. M., Martin S. C., Degioia-Eastwood K., 1993, AJ, 105, 1822
Damiani F. et al., 2016, A\&A, 591, A74

Damiani F. et al., 2017, A\&A, 603, A81

Davidson K., Humphreys R. M., 1997, ARA\&A, 35, 1

Davidson K., Smith N., Gull T. R., Ishibashi K., Hillier D. J., 2001, AJ, 121, 1569

de Bruijne J. H. J., 1999, MNRAS, 310, 585

de Graauw T., Lidholm S., Fitton B., Beckman J., Israel F. P., Nieuwenhuijzen H., Vermue J., 1981, A\&A, 102, 257

Deharveng L., Maucherat M., 1975, A\&A, 41, 27

Dickel H. R., 1974, A\&A, 31, 11

Doran E. I. et al., 2013, A\&A, 558, A134

Drilling J. S., Landolt A. U., 2000, in Cox A. N., ed., Allen's Astrophysical Quantities. Springer, Berlin, p. 381

Efremov Y. N., Elmegreen B. G., 1998, MNRAS, 299, 643

Feast M. W., Thackeray A. D., Wesselink A. J., 1957, Mem. R. Astron. Soc., 68,1

Feigelson E. D. et al., 2011, ApJS, 194, 9

Feinstein A., Marraco H. G., Muzzio J. C., 1973, A\&AS, 12, 331

Feinstein A., Marraco H. G., Forte J. C., 1976, A\&AS, 24, 389

Forte J. C., 1978, AJ, 83, 1199

Fullerton A. W., Gies D. R., Bolton C. T., 1996, ApJS, 103, 475

Fullerton A. W., Massa D. L., Prinja R. K., Owocki S. P., Cranmer S. R., 1997, A\&A, 327, 699

Gaczkowski B., Preibisch T., Ratzka T., Roccatagliata V., Ohlendorf H., Zinnecker H., 2013, A\&A, 549, A67

Gagné M. et al., 2011, ApJS, 194, 5

Gaia Collaboration et al., 2016a, A\&A, 595, A1

Gaia Collaboration et al., 2016b, A\&A, 595, A2

Gamen R. et al., 2006, A\&A, 460, 777

García B., Malaroda S., Levato H., Morrell N., Grosso M., 1998, PASP, 110,53

Gardner F. F., Milne D. K., Mezger P. G., Wilson T. L., 1970, A\&A, 7, 349

Garmany C. D., Conti P. S., Massey P., 1980, ApJ, 242, 1063

Gieles M., 2013, presentation at 'Massive Stars: From alpha to Omega', p. 7. Available at: http://articles.adsabs.harvard.edu/cgibin/get_file?pdfs/msao./2013/2013msao.confE...7G.pdf (accessed May 17, 2017)

Gieles M., Sana H., Portegies Zwart S. F., 2010, MNRAS, 402, 1750

Gies D. R., Bolton C. T., 1986, ApJS, 61, 419

Hénault-Brunet V. et al., 2012, A\&A, 546, A73

Herbst W., 1976, ApJ, 208, 923

Hills J. G., 1980, ApJ, 235, 986

Högbom J. A., 1974, A\&AS, 15, 417

Huang W., Gies D. R., 2006, ApJ, 648, 580

Huchtmeier W. K., Day G. A., 1975, A\&A, 41, 153

Humphreys R. M., 1973, A\&AS, 9, 85

Hur H., Sung H., Bessell M. S., 2012, AJ, 143, 41

Iglesias-Marzoa R., López-Morales M., Jesús Arévalo Morales M., 2015, PASP, 127, 567

Kenyon S. J., Hartmann L., 1995, ApJS, 101, 117

Kharchenko N. V., 2001, Kinematika i Fizika Nebesnykh Tel, 17, 409

Kharchenko N. V., Roeser S., 2009, VizieR Online Data Catalog, 1280

Kiminki D. C., Kobulnicky H. A., 2012, ApJ, 751, 4

Kiminki D. C. et al., 2007, ApJ, 664, 1102

Kiminki D. C. et al., 2008, ApJ, 681, 735

Kiminki M. M., Smith N., Reiter M., Bally J., 2017, MNRAS, 468, 2469

Kobulnicky H. A. et al., 2014, ApJS, 213, 34

Kramida A., Ralchenko Y., Reader J., NIST ASD Team 2016, NIST Atomic Spectra Database (version 5.4), Available at: http://physics.nist.gov/asd (last accessed January 14, 2018)

Kumar B., Sharma S., Manfroid J., Gosset E., Rauw G., Nazé Y., Kesh Yadav R., 2014, A\&A, 567, A109

Lada C. J., Lada E. A., 1991, in Janes K., ed., ASP Conf. Ser. Vol. 13, The Formation and Evolution of Star Clusters. Astron. Soc. Pac., San Francisco, p. 3

Lada C. J., Lada E. A., 2003, ARA\&A, 41, 57

Levato H., García B., Loustó C., Morrell N., Saizar P., 1986, Rev. Mex. Astron. Astrofis., 13, 3 
Levato H., Malaroda S., Garcia B., Morrell N., Solivella G., 1990, ApJS, 72,323

Levato H., Malaroda S., Morrell N., Garcia B., Hernandez C., 1991a, ApJS, 75,869

Levato H., Malaroda S., Garcia B., Morrell N., Solivella G., Grosso M., 1991b, Ap\&SS, 183, 147

Lindegren L. et al., 2016, A\&A, 595, A4

Maíz Apellániz J. et al., 2013, presentation at 'Massive Stars: From alpha to Omega'. preprint (arXiv:1306.6417)

Maíz Apellániz J. et al., 2016, ApJS, 224, 4

Markwardt C. B., 2009, in Bohlender D. A., Durand D., Dowler P., eds, ASP Conf. Ser. Vol. 411, Astronomical Data Analysis Software and Systems XVIII. Astron. Soc. Pac., San Francisco, p. 251

Martins F., Schaerer D., Hillier D. J., 2005, A\&A, 436, 1049

Martins F., Marcolino W., Hillier D. J., Donati J.-F., Bouret J.-C., 2015, A\&A, 574, A142

Mason B. D., Hartkopf W. I., Gies D. R., Henry T. J., Helsel J. W., 2009, AJ, 137, 3358

Massey P., 1980, ApJ, 236, 526

Massey P., Conti P. S., 1981, ApJ, 244, 173

Massey P., Johnson J., 1993, AJ, 105, 980

Massey P., Thompson A. B., 1991, AJ, 101, 1408

Massey P., DeGioia-Eastwood K., Waterhouse E., 2001, AJ, 121, 1050

Mayer P., Lorenz R., Drechsel H., Abseim A., 2001, A\&A, 366, 558

McClure-Griffiths N. M., Dickey J. M., 2007, ApJ, 671, 427

Megeath S. T., Cox P., Bronfman L., Roelfsema P. R., 1996, A\&A, 305, 296

Moe M., Di Stefano R., 2017, ApJS, 230, 15

Moffat A. F. J., 1978, A\&A, 68, 41

Moffat A. F. J., Seggewiss W., 1978, A\&A, 70, 69

Moffat A. F. J., Seggewiss W., 1979, A\&A, 77, 128

Mohr-Smith M. et al., 2017, MNRAS, 465, 1807

Morrell N., Garcia B., Levato H., 1988, PASP, 100, 1431

Morrell N. I. et al., 2001, MNRAS, 326, 85

Morrison N. D., Conti P. S., 1980, ApJ, 239, 212

Munoz M., Moffat A. F. J., Hill G. M., Shenar T., Richardson N. D., Pablo H., St-Louis N., Ramiaramanantsoa T., 2017, MNRAS, 467, 3105

Nazé Y., Antokhin I. I., Sana H., Gosset E., Rauw G., 2005, MNRAS, 359, 688

Niemela V. S., Moffat A. F. J., 1982, ApJ, 259, 213

Niemela V. S., Massey P., Conti P. S., 1980, ApJ, 241, 1050

Niemela V. S., Morrell N. I., Fernández Lajús E., Barbá R., Albacete Colombo J. F., Orellana M., 2006, MNRAS, 367, 1450

Oort J. H., Spitzer L., Jr, 1955, ApJ, 121, 6

Otero S. A., 2006, Open Eur. J. Var. Stars, 45, 1

Penny L. R., Gies D. R., Hartkopf W. I., Mason B. D., Turner N. H., 1993, PASP, 105, 588

Pojmanski G., 1997, AcA, 47, 467

Povich M. S. et al., 2011a, ApJS, 194, 6

Povich M. S. et al., 2011b, ApJS, 194, 14

Preibisch T., Mamajek E., 2008, in Reipurth B., ed., Handbook of Star Forming RegionsVolume II. Astron. Soc. Pac., San Francisco, CA, p. 235

Preibisch T., Schuller F., Ohlendorf H., Pekruhl S., Menten K. M., Zinnecker H., 2011, A\&A, 525, A92

Prša A., Zwitter T., 2005, ApJ, 628, 426

Rathborne J. M., Brooks K. J., Burton M. G., Cohen M., Bontemps S., 2004, A\&A, 418, 563

Rauw G., Vreux J.-M., Gosset E., Hutsemekers D., Magain P., Rochowicz K., 1996, A\&A, 306, 771

Rauw G., Sana H., Gosset E., Vreux J.-M., Jehin E., Parmentier G., 2000, A\&A, 360, 1003

Rauw G., Sana H., Antokhin I. I., Morrell N. I., Niemela V. S., Albacete Colombo J. F., Gosset E., Vreux J.-M., 2001, MNRAS, 326, 1149

Rauw G., Nazé Y., Fernández Lajús E., Lanotte A. A., Solivella G. R., Sana H., Gosset E., 2009, MNRAS, 398, 1582

Rebolledo D. et al., 2016, MNRAS, 456, 2406

Ritchie B. W., Clark J. S., Negueruela I., Crowther P. A., 2009, A\&A, 507, 1585
Roberts D. H., Lehar J., Dreher J. W., 1987, AJ, 93, 968

Rochau B., Brandner W., Stolte A., Gennaro M., Gouliermis D., Da Rio N., Dzyurkevich N., Henning T., 2010, ApJ, 716, L90

Rochau B. et al., 2011, MNRAS, 418, 949

Sabín-Sanjulián C. et al., 2014, A\&A, 564, A39

Sana H. et al., 2012, Science, 337, 444

Sana H. et al., 2013, A\&A, 550, A107

Sana H. et al., 2014, ApJS, 215, 15

Schnurr O., Moffat A. F. J., St-Louis N., Morrell N. I., Guerrero M. A., 2008, MNRAS, 389, 806

Schweickhardt J., Schmutz W., Stahl O., Szeifert T., Wolf B., 1999, A\&A, 347,127

Sexton R. O., Povich M. S., Smith N., Babler B. L., Meade M. R., Rudolph A. L., 2015, MNRAS, 446, 1047

Smith R. G., 1987, MNRAS, 227, 943

Smith N., 2004, MNRAS, 351, L15

Smith N., 2006, MNRAS, 367, 763

Smith N., Brooks K. J., 2007, MNRAS, 379, 1279

Smith N., Brooks K. J., 2008, in Reipurth B., ed., Handbook of Star Forming Regions, Volume II. Astron. Soc. Pac., San Francisco, CA, p. 138

Smith N., Conti P. S., 2008, ApJ, 679, 1467

Smith N., Stassun K. G., 2017, AJ, 153, 125

Smith N., Egan M. P., Carey S., Price S. D., Morse J. A., Price P. A., 2000, ApJ, 532, L145

Smith N., Bally J., Brooks K. J., 2004, AJ, 127, 2793

Smith N., Stassun K. G., Bally J., 2005, AJ, 129, 888

Smith N., Bally J., Walborn N. R., 2010a, MNRAS, 405, 1153

Smith N. et al., 2010b, MNRAS, 406, 952

Solivella G. R., Niemela V. S., 1999, in Morrell N. I., Niemela V. S., Barbá R. H., eds, Revista Mexicana de Astronomia y Astrofisica Conference Series Vol. 8, Workshop on Hot Stars in Open Clusters of the Galaxy and the Magellenic Clouds. Universidad Nacional Autónoma de México, Mexico, p. 145

Sota A., Maíz Apellániz J., Morrell N. I., Barbá R. H., Walborn N. R., Gamen R. C., Arias J. I., Alfaro E. J., 2014, ApJS, 211, 10

Soubiran C., Jasniewicz G., Chemin L., Crifo F., Udry S., Hestroffer D., Katz D., 2013, A\&A, 552, A64

Spitzer L., 1978, Physical Processes in the Interstellar Medium. WileyInterscience, New York, NY

Steenbrugge K. C., de Bruijne J. H. J., Hoogerwerf R., de Zeeuw P. T., 2003 , A\&A, 402, 587

Tapia M., Roth M., Marraco H., Ruiz M. T., 1988, MNRAS, 232, 661

Tapia M., Roth M., Vázquez R. A., Feinstein A., 2003, MNRAS, 339, 44

Thackeray A. D., Tritton S. B., Walker E. N., 1973, Mem. R. Astron. Soc., 77,199

Thé P. S., Vleeming G., 1971, A\&A, 14, 120

Thé P. S., Bakker R., Antalova A., 1980a, A\&AS, 41, 93

Thé P. S., Bakker R., Tjin A Djie H. R. E., 1980b, A\&A, 89, 209

Tokovinin A., Fischer D. A., Bonati M., Giguere M. J., Moore P., Schwab C., Spronck J. F. P., Szymkowiak A., 2013, PASP, 125, 1336

Tovmassian H. M., Hovhannessian R. K., Epremian R. A., 1994, Ap\&SS, 213,175

Turner D. G., Moffat A. F. J., 1980, MNRAS, 192, 283

Tutukov A. V., 1978, A\&A, 70, 57

Vallée J. P., 2014, AJ, 148, 5

Vazquez R. A., Baume G., Feinstein A., Prado P., 1996, A\&AS, 116, 75

Walborn N. R., 1973, ApJ, 179, 517

Walborn N. R., 1982a, AJ, 87, 1300

Walborn N. R., 1982b, ApJ, 254, L15

Walborn N. R., 1995, in Niemela V., Morrell N., Feinstein A., eds, Revista Mexicana de Astronomia y Astrofisica Conference Series Vol. 2, The Eta Carinae Region: A Laboratory of Stellar Evolution. Universidad Nacional Autónoma de México, Mexico, p. 51

Walborn N. R., 2009, in Livio M., Villaver E., eds, Space Telescope Science Institute Symposium Series Vol. 20, Massive Stars: From Pop III and GRBs to the Milky Way. Cambridge Uni. Press, Cambridge, UK, p. 167

Walborn N. R., Hesser J. E., 1975, ApJ, 199, 535

Walborn N. R., Liller M. H., 1977, ApJ, 211, 181 
Walborn N. R. et al., 2002a, AJ, 123, 2754

Walborn N. R., Danks A. C., Vieira G., Landsman W. B., 2002b, ApJS, 140, 407

Walborn N. R., Smith N., Howarth I. D., Vieira Kober G., Gull T. R., Morse J. A., 2007, PASP, 119, 156

Walborn N. R. et al., 2014, A\&A, 564, A40

Walsh J. R., 1984, A\&A, 138, 380

Wang J. et al., 2011, ApJS, 194, 11

Whiteoak J. B. Z., 1994, ApJ, 429, 225

Williams S. J., Gies D. R., Hillwig T. C., McSwain M. V., Huang W., 2011, AJ, 142, 146

Wright N. J., Drew J. E., Mohr-Smith M., 2015, MNRAS, 449, 741

Wright N. J., Bouy H., Drew J. E., Sarro L. M., Bertin E., Cuillandre J.-C., Barrado D., 2016, MNRAS, 460, 2593

Wu Z.-Y., Zhou X., Ma J., Du C.-H., 2009, MNRAS, 399, 2146

Yonekura Y., Asayama S., Kimura K., Ogawa H., Kanai Y., Yamaguchi N., Barnes P. J., Fukui Y., 2005, ApJ, 634, 476

\section{SUPPORTING INFORMATION}

Supplementary data are available at MNRAS online.

\section{tab2.txt}

Please note: Oxford University Press is not responsible for the content or functionality of any supporting materials supplied by the authors. Any queries (other than missing material) should be directed to the corresponding author for the article.

\section{APPENDIX A：PERIODOGRAMS}

Here we present frequency power spectra for the binary systems fit in Section 3. We use the discrete Fourier transform (DFT) and one-dimensional CLEAN algorithm ${ }^{4}$ of Roberts et al. (1987) as implemented in IDL by A. W. Fullerton (see Fullerton et al. 1997). The DFT is computed up to a maximum frequency of $f_{\max }=1 /\left(2 \Delta t_{\min }\right)$, where $\Delta t_{\min }$ is the minimum time interval between observations. CLEAN deconvolves the window function from the DFT, removing aliases introduced by uneven time sampling. We run CLEAN for 100 iterations with a gain of 0.5 .

The resulting periodograms are used first to check for periodicity and second to corroborate the periods found by the orbital fitting package RVFIT (Iglesias-Marzoa et al. 2015) for HD 92607, HD 93576, and HDE 305536. RVFIT does not require an initial estimate of the period, and we leave the period as a free parameter when fitting these three systems. For the eclipsing binary HDE 303312, we fix the period in RVFIT at the photometric period as described in Section 3.3.

The periodograms for the primary and secondary components of HD 92607 (Fig. A1) both have clear peaks at frequencies of $\approx 0.276 \mathrm{~d}^{-1}$, corresponding to a period of $\sim 3.6 \mathrm{~d}$. The full, unconstrained fit with RVFIT (see Section 3.1) finds a period of $3.6993 \mathrm{~d}$. Visual inspection of phase-folded RV curves rules out most common possible alias periods. We cannot definitively exclude the $1+f$ alias, which is above our maximum searchable frequency and corresponds to a period of $\sim 0.79 \mathrm{~d}$; however, a subday period would be extremely unusual for a main-sequence $\mathrm{O}+\mathrm{O}$ binary (Kiminki \& Kobulnicky 2012; Sana et al. 2012; Almeida et al. 2017; Moe \& Di Stefano 2017).

\footnotetext{
${ }^{4}$ The two-dimensional CLEAN algorithm was first developed by Högbom
} (1974).

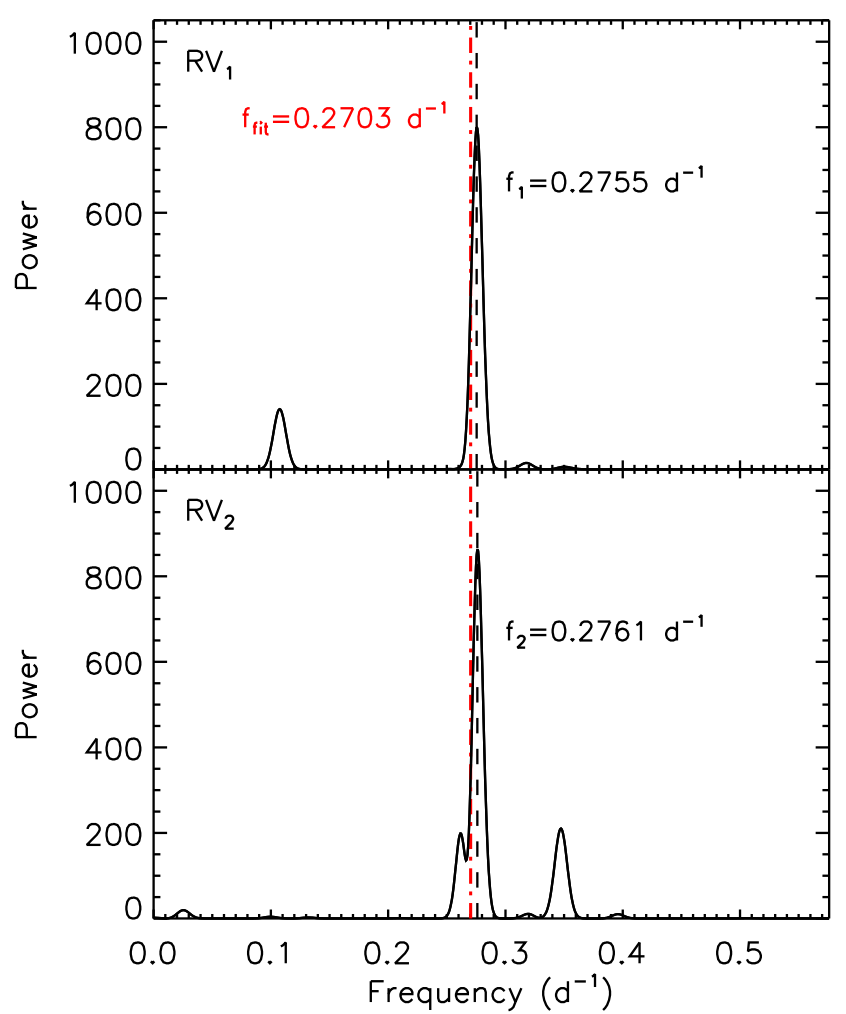

Figure A1. CLEANed power spectra of the primary (top) and secondary (bottom) RV components of the SB2 HD 92607. The black dashed lines mark the frequencies $f_{1}$ and $f_{2}$ corresponding to the strongest peak in each power spectrum. The red dot-dashed line in both panels indicates the frequency $f_{\text {fit }}$ corresponding to the final best-fitting orbital period from an unconstrained simultaneous fit to both components with RVFIT.

The components of HD 92607 are of very similar spectral type (O8.5 V + O9 V; Sexton et al. 2015). If we swap the values of $\mathrm{RV}_{1}$ and $\mathrm{RV}_{2}$ at several epochs, the periodograms of both components show clear peaks at $0.538 \mathrm{~d}^{-1}$, or a period of $1.859 \mathrm{~d}$. With these component assignments, RVFIT finds a plausible solution for a similar period of $1.84957 \mathrm{~d}$. This fit requires a substantial eccentricity $(e=0.35)$ and an approximate inclination of $45^{\circ}$.

The periodogram derived from our CHIRON data for HD 93576 (upper panel in Fig. A2) shows a single strong peak at a frequency of $0.543 \mathrm{~d}^{-1}$, or a period of $1.84 \mathrm{~d}$. A similar peak appears when this system's data from Levato et al. (1990) are included (lower panel of Fig. A2). The full, unconstrained fit with RVFIT to the combined data set (see Section 3.2) finds a period of $1.852102 \mathrm{~d}$. Before CLEANing, the DFT for HD 93576 also shows a peak at the $1-f$ alias of $0.460 \mathrm{~d}^{-1}$, or a period of $2.174 \mathrm{~d}$. We can force a plausible solution at this period in RVFIT if we constrain the range of allowed periods and fit only to our CHIRON data; the combined data set including Levato et al. (1990) RVs is incompatible with this period. As this alias peak is removed by the CLEAN deconvolution, it appears to be an artefact of our observational time sampling.

The RV periodogram for the eclipsing binary HDE 303312 (Fig. A3) has several peaks. The strongest peak is at a frequency of $0.503 \mathrm{~d}^{-1}$ or a period of $1.99 \mathrm{~d}$. This period - which, as a nearinteger multiple of days, is likely a sampling artefact - is ruled out by visual inspection of the phase-folded RV curve and by constrained tests in RVFIT. The second-highest peak in the periodogram is at a frequency of $0.114 \mathrm{~d}^{-1}$, or a period of $\sim 8.8 \mathrm{~d}$. Unconstrained fits 


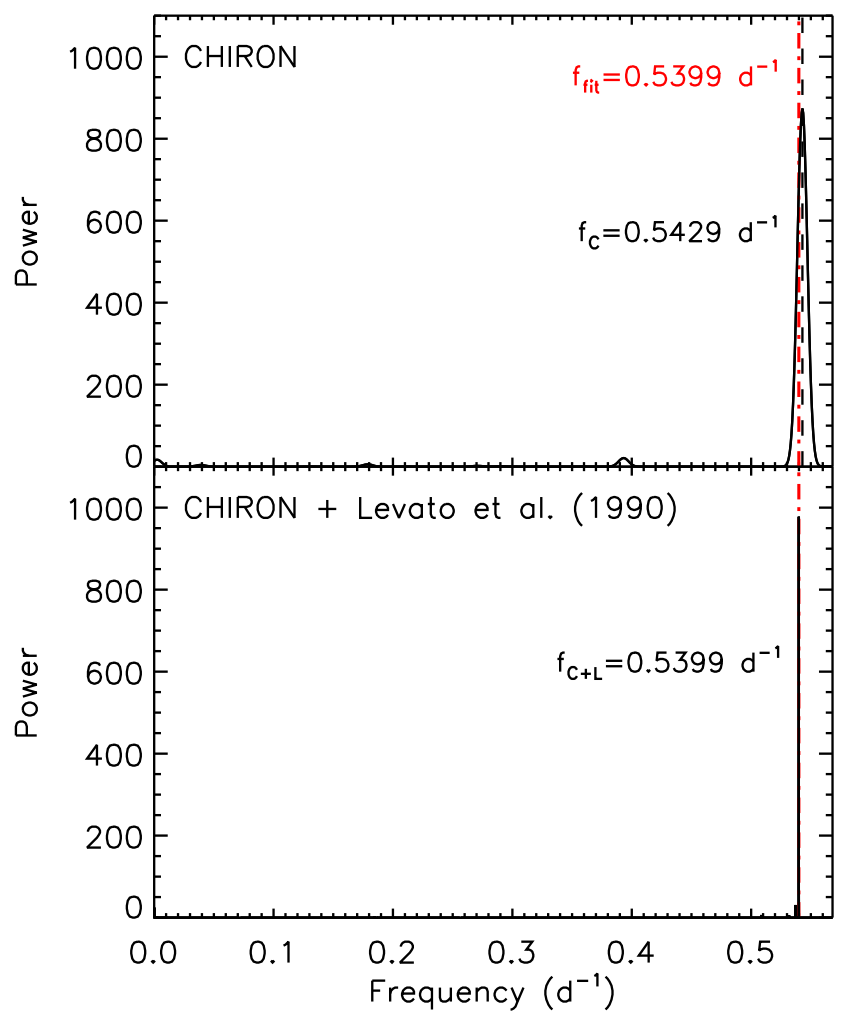

Figure A2. Top: CLEANed power spectrum of our CHIRON RV data for the SB1 HD 93576. The black dashed line marks the frequency $f_{\mathrm{C}}$ corresponding to the strongest peak. Bottom: as above, including RV data from Levato et al. (1990). The frequency $f_{\mathrm{C}+\mathrm{L}}$ corresponds to the strongest peak. The red dotdashed line in both panels indicates the frequency $f_{\text {fit }}$ corresponding to the final best-fitting orbital period from an unconstrained fit to the combined data set with RVFIT.

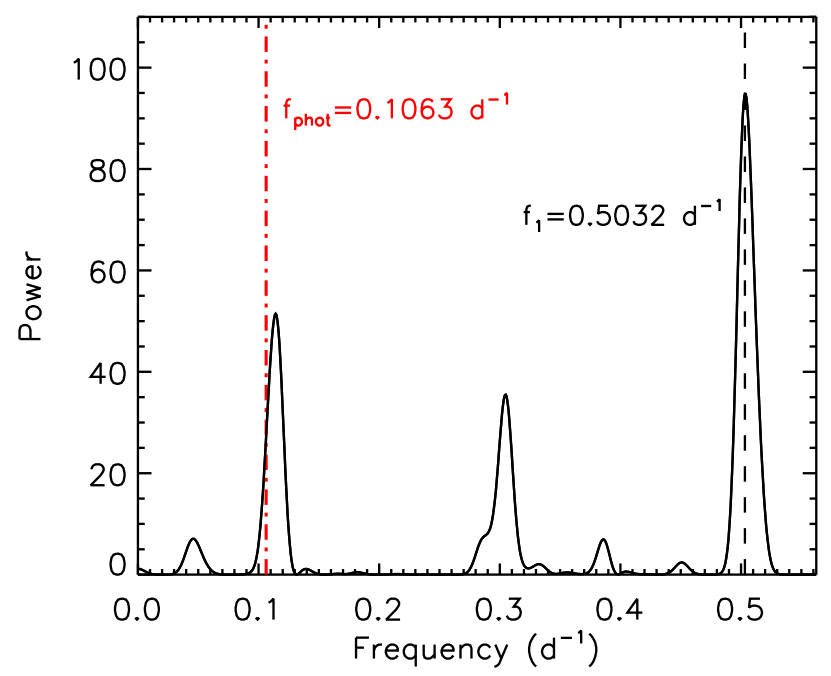

Figure A3. CLEANed power spectrum of our CHIRON RV data for the eclipsing SB1 HDE 303312. The black dashed line marks the frequency $f_{1}$ corresponding to the strongest peak. The red dot-dashed line indicates the frequency $f_{\text {phot }}$ of the ASAS $V$-band light curve.

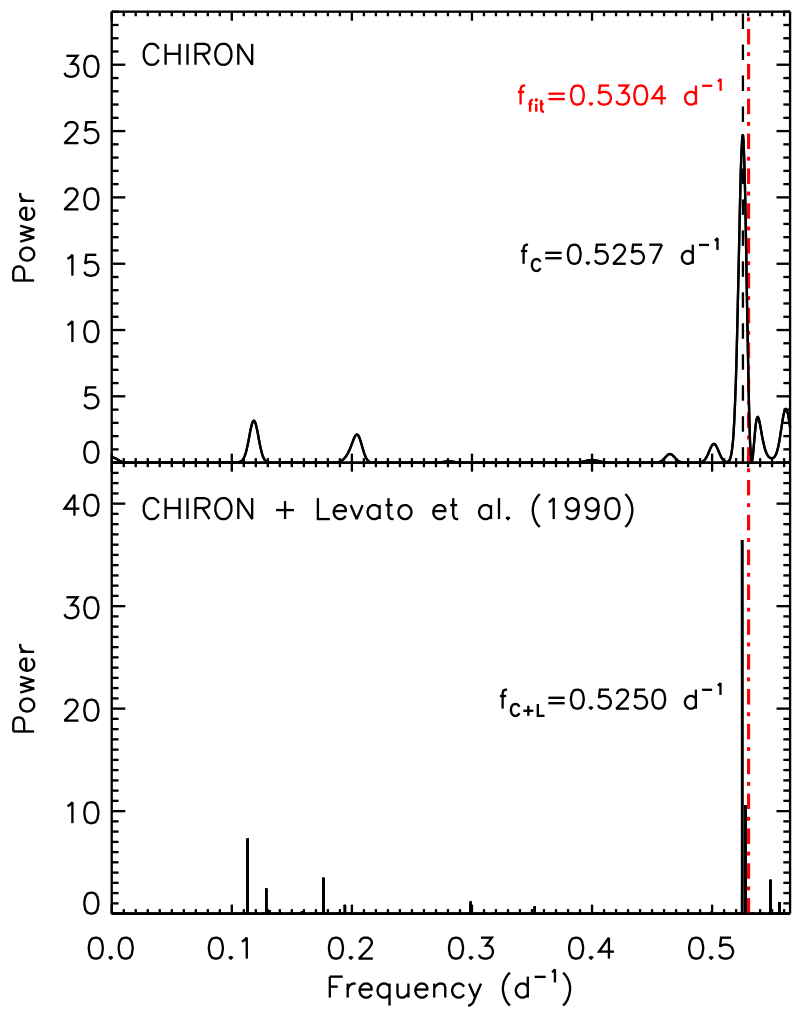

Figure A4. Top: CLEANed power spectrum of our CHIRON RV data for the SB1 HDE 305536. The black dashed line marks the frequency $f_{\mathrm{C}}$ corresponding to the strongest peak. Bottom: as above, including RV data from Levato et al. (1990). The frequency $f_{\mathrm{C}+\mathrm{L}}$ corresponds to the strongest peak. The red dot-dashed line in both panels indicates the frequency $f_{\text {fit }}$ corresponding to the final best-fitting orbital period from an unconstrained fit to our CHIRON data with RVFIT.

in RVFIT converge on a period between 9.41 and $9.65 \mathrm{~d}$, close to the photometric period of $9.4111 \mathrm{~d}$ (which corresponds to a frequency of $0.10626 \mathrm{~d}^{-1}$ ). As described in Section 3.3, we fix the period at the photometric period for subsequent fitting.

The periodogram derived from our CHIRON data for HDE 305536 (top panel of Fig. A4) has its highest peak at a frequency of $0.526 \mathrm{~d}^{-1}$ or a period of $1.90 \mathrm{~d}$. A similar peak frequency is found when this system's data from Levato et al. (1990) are included (bottom panel of Fig. A4). Visual inspection of phase-folded RV curves and constrained tests in RVFIT rule out common possible aliases. The full, unconstrained fit to our CHIRON data with RVFIT finds an orbital period of $1.88535 \mathrm{~d}$, corresponding to a frequency of $0.53041 \mathrm{~d}^{-1}$. As described in Section 3.4, RVFIT is unable to converge on a solution that includes the Levato et al. (1990) data.

This paper has been typeset from a $\mathrm{T}_{\mathrm{E}} \mathrm{X} / \mathrm{L} \mathrm{T}_{\mathrm{E}} \mathrm{X}$ file prepared by the author. 